\title{
Chemically Stable Polyarylether-Based Metallophthalocyanine Frameworks with High Carrier Mobilities for Capacitive Energy Storage
}

Chongqing Yang, ${ }^{1}$ Kaiyue Jiang, ${ }^{2}$ Qi Zheng, ${ }^{3}$ Xinle Li, ${ }^{1,3,4}$ Haiyan Mao, ${ }^{5}$ Wenkai Zhong, ${ }^{6,7}$ Cheng Chen, ${ }^{2}$ Bing Sun, ${ }^{1,8}$ Haimei Zheng, ${ }^{3}$ Xiaodong Zhuang, ${ }^{2}$ Jeffrey A. Reimer, ${ }^{3,5}$ Yi Liu, ${ }^{1,3}$ and Jian Zhang $^{1 *}$

1. The Molecular Foundry, Lawrence Berkeley National Laboratory, Berkeley, California 94720, United States

2. School of Chemistry and Chemical Engineering, Shanghai Jiao Tong University, Shanghai 200240, P.R. China

3. Materials Sciences Division, Lawrence Berkeley National Laboratory, Berkeley, California 94720, United States

4. Department of Chemistry, Clark Atlanta University, Atlanta, Georgia 30314, United States

5. Department of Chemical and Biomolecular Engineering, University of California, Berkeley, California 94720, United States

6. Advanced Light Source, Lawrence Berkeley National Laboratory, Berkeley, CA 94720, United States

7. Frontiers Science Center for Transformative Molecules, In-situ Center for Physical Science, and Center of Hydrogen Science, School of Chemistry and Chemical Engineering, Shanghai Jiao Tong University, Shanghai 200240, China

8. School of Science, China University of Geosciences (Beijing), Beijing 100083, P.R. China

${ }^{*}$ Corresponding author 


\section{Experimental section.}

\section{Materials}

All starting materials were purchased from commercial suppliers and directly used without further purification. All synthetic reactions were performed by Schleck techniques under nitrogen atmosphere or vacuum conditions. Column chromatography was applied by silica gel.

\section{Monomer synthesis}

The synthesis of $(2,3,9,10,16,17,23,24$-octahydroxylphthalocyaninato $)$ metal ${ }^{\mathrm{II}}\left(\mathrm{PcMOH}_{8}, \mathrm{M}=\mathrm{Zn}, \mathrm{Cu}, \mathrm{Ni}\right.$ and $\left.\mathrm{Co}\right)$ were carried out according to previous reported methods. ${ }^{1-3} \mathrm{PcZnOH}_{8}$ was prepared as a model compound to confirm the structure of obtained $\mathrm{PcMOH}_{8}$.

(2,3,9,10,16,17,23,24-octahydroxylphthalocyaninato) zinc ${ }^{\mathrm{II}}$ (PcZnOH8): ${ }^{1} \mathrm{H}$ NMR (ds-pyridine): $\delta$ (ppm) 9.56 $(s, 8 \mathrm{H}), 6.82(s, 8 \mathrm{H})$. Solid State ${ }^{13} \mathrm{C}$ ssNMR: $\delta(\mathrm{ppm})$ 110, 124, 131, 148. MALDI-TOF MS for $\mathrm{C}_{32} \mathrm{H}_{16} \mathrm{~N}_{8} \mathrm{O}_{8} \mathrm{Zn}(\mathrm{calcd}$. 704.04): found 704.13.

(2,3,9,10,16,17,23,24-octahydroxylphthalocyaninato) nickel ${ }^{\mathrm{II}}$ (PcNiOH$\left.)_{8}\right){ }^{1} \mathrm{H}$ NMR $\left(d_{6}-D M S O\right): \delta(\mathrm{ppm}) 10.37$ $(s, 8 \mathrm{H}), 8.58(s, 8 \mathrm{H})$. MALDI-TOF MS for $\mathrm{C}_{32} \mathrm{H}_{16} \mathrm{~N}_{8} \mathrm{O}_{8} \mathrm{Ni}$ (calcd. 698.04): found 698.17.

(2,3,9,10,16,17,23,24-octahydroxylphthalocyaninato) copper $^{\mathrm{II}} \quad$ (PcCuOH $\left._{8}\right): \quad$ MALDI-TOF $\quad$ MS for $\mathrm{C}_{32} \mathrm{H}_{16} \mathrm{~N}_{8} \mathrm{O}_{8} \mathrm{Cu}$ (calcd. 703.04): found 703.13.

(2,3,9,10,16,17,23,24-octahydroxylphthalocyaninato) cobalt $^{\mathrm{II}} \quad$ (PcCoOH$\left._{8}\right): \quad$ MALDI-TOF $\quad$ MS for $\mathrm{C}_{32} \mathrm{H}_{16} \mathrm{~N}_{8} \mathrm{O}_{8} \mathrm{Co}$ (calcd. 699.04): found 699.15.

\section{Synthesis of PAE-PcM powder}

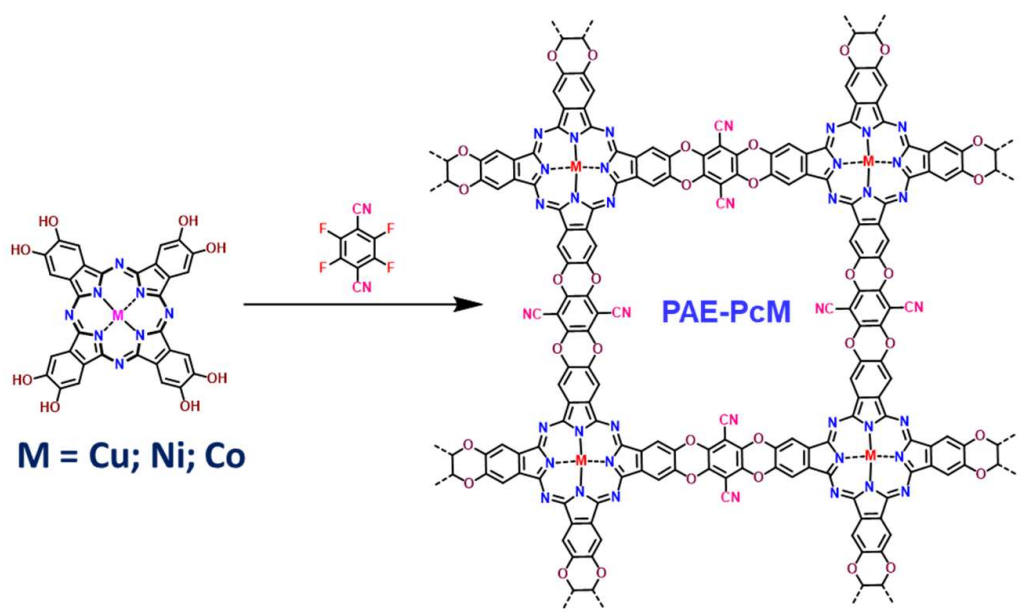

Schematic illustration for the synthesis of PAE-PcM $(\mathrm{M}=\mathrm{Cu}, \mathrm{Ni}$ and $\mathrm{Co})$.

PAE-PcCu: A $5 \mathrm{~mL}$ microwave Biotage vial was charged with $\mathrm{PcCuOH}_{8}(40 \mathrm{mg}), \mathrm{TFTPN}(22.8 \mathrm{mg}), \mathrm{K}_{2} \mathrm{CO}_{3}(60 \mathrm{mg})$ 
powders and mixed solvents (DMAc/Mesitylene $1.2 \mathrm{~mL} / 1.2 \mathrm{~mL}$ ) under $\mathrm{N}_{2}$ atmosphere. The obtained mixture was sonicated for $10 \mathrm{~min}$ and degassed by the freeze-pump-thaw method for 3 times. After being sealed under vacuum, the whole vial was heated at $120^{\circ} \mathrm{C}$ for 5 days. Then the whole mixture was centrifuged and washed by DMAc, water and acetone for 2 times, respectively. Finally, the solid was immersed in DMAc, acetone, THF and hexane each for 1 days. The resulted dark blue powders were collected for further characterization. Yield: 56\%.

PAE-PcNi: The PAE-PcNi was synthesized similarly with PAE-PcCu. Yield: 48\%.

PAE-PcCo: A $5 \mathrm{~mL}$ microwave Biotage vial was charged with $\mathrm{PcCoOH}_{8}(40 \mathrm{mg})$, TFTPN (22.8 mg), $\mathrm{K}_{2} \mathrm{CO}_{3}(60 \mathrm{mg})$ powders and mixed solvents (DMAc/Mesitylene $1.4 \mathrm{~mL} / 1.0 \mathrm{~mL}$ ) under $\mathrm{N}_{2}$ atmosphere. The obtained mixture was sonicated for $10 \mathrm{~min}$ and degassed by the freeze-pump-thaw method for 3 times. After being sealed under vacuum, the whole vial was heated at $140{ }^{\circ} \mathrm{C}$ for 5 days. Then the whole mixture was centrifuged and washed by DMAc, water, and acetone for 2 times, respectively. Finally, the solid was immersed in DMAc, acetone, THF and hexane each for 1 days. The resulted dark blue powders were collected for further characterization. Yield: 55\%.

\section{Preparation of PAE-PcM films}

The preparation of PAE-PcM thin films were conducted through the solvothermal method. $15 \mathrm{mg} \mathrm{PcMOH}_{8}, 8.4 \mathrm{mg}$ TFTPN and $15 \mathrm{mg} \mathrm{NaOAc}$ were added into a polytetrafluoroethylene (PTFE) autoclave with $12 \mathrm{~mL}$ THF and $4 \mathrm{~mL}$ DMAc. The whole mixture was bubbled with $\mathrm{N}_{2}$ for $5 \mathrm{~min}$. Then homemade $\Pi$-shaped PTFE holder with different kinds of substrates $\left(\mathrm{Si} / \mathrm{SiO}_{2}\right.$; ITO; quatz etc.) were slowly immersed into the above solution mixture. After sealed the autoclave, the whole steel container was heated at $120^{\circ} \mathrm{C}$ (for PAE-PcCu and PAE-PcNi thin films) and $140{ }^{\circ} \mathrm{C}$ (for PAE-PcCo thin films) respectively. When cooling down to room temperature, the obtained substrates were washed with acetone and water respectively. Then the substrates were immersed in dry THF for $12 \mathrm{~h}$ and dried before further characterization.

\section{Iodine doping of PAE-PcCu film}

The iodine doping was performed like the previous reported method. ${ }^{4-5} \mathrm{In}$ a sealed $20 \mathrm{~mL}$ vial, the PAE-PcCu film with gold electrode was exposed in $\mathrm{I}_{2}$ vapor at room temperature for $12 \mathrm{~h}$. Then the device was brought out for the conductivity measurement directly.

\section{Instrumentation and characterization}

The solution based ${ }^{1} \mathrm{H}$ and ${ }^{13} \mathrm{C}$ NMR spectra were recorded on AVANCE II 500 (Bruker, Germany) using the deuterated solvents ( $d_{6}$-DMSO, $d_{5}$-pyridine) with the tetramethylsilane as the internal reference. The solid-state ${ }^{13} \mathrm{C}$ cross-polarization magnetic angle spinning (CP-MAS) NMR spectra were recorded on the AVANCE II 500 (Bruker, Germany) at a magnetic field strength of $11.7 \mathrm{~T}$. The ${ }^{13} \mathrm{C}$ cross-polarization contact time was $3 \mathrm{~ms}$ with high power ${ }^{1} \mathrm{H}$ decoupling. MAS experiments were performed on 4-mm MAS probes at a spinning speed of $10 \mathrm{kHz}$. The ${ }^{13} \mathrm{C}$ signals was referenced to the methylene signal of adamantane at $38.5 \mathrm{ppm}$. MALDI-TOF spectrometry analysis was performed on a 4800 MALDI TOF/TOF analyzer. Fourier transform infrared (FTIR) spectra were recorded on the Nicolet $^{\mathrm{TM}}$ iS50 FT-IR system (Thermo Fisher, USA). Powder X-ray diffraction (PXRD) patterns were recorded on a Rigaku X-ray diffractometer using $\mathrm{Cu}-\mathrm{K} \alpha$ radiation $(\lambda=0.15418 \mathrm{~nm})$ at $40 \mathrm{kV}$ and $20 \mathrm{~mA}$. Wide-angle $\mathrm{X}$-ray 
scattering patterns (in transmission mode), grazing-incident wide-angle X-ray scattering patterns (incident angle $0.16^{\circ}$ ) and O-XANES were obtained at Advanced Light Source beamline 7.3.3 in LBNL. O K-edge XANES measurements were performed at beamline 11.0.1.2 of Advanced Light Source, LBNL. The samples were attached on carbon tapes for the XANES measurements with total electron yield (TEY) mode. Normalization of the XANES spectra was performed with ATHENA package. Nitrogen sorption measurements were conducted at $77 \mathrm{~K}$ on an ASAP 2020 sorption analyzer (Micromeritics Instrument Co., USA) by immersing tubes into liquid nitrogen. The corresponding specific surface area were calculated by the Brunauer-Emmett-Teller (BET) method, and the pore size distribution were estimated from the adsorption isotherm by the quenched solid density functional theory (QSDFT) equilibrium model. The X-ray photoelectron spectroscopy (XPS) spectra were obtained using a K-Alpha XPS apparatus equipped with a monochromatic Al K( $\alpha$ ) source (Thermo Fisher Scientific, USA).. The scanning electron microscopy (SEM) and the transmission electron microscopy (TEM) images were recorded on a field emission scanning electron microscope (FESEM, Zeiss Gemini 500) and a high-resolution transmission electron microscope (HRTEM, JEM-2100, JEOL, Japan), respectively. The atomic force microscopy (AFM) images were recorded on a Brucker Dimension Icon AFM with tapping mode. Hall-effect measurement was recorded on an Ecopia HMS-3000 Hall Measurement System.

\section{Electronic structure calculation}

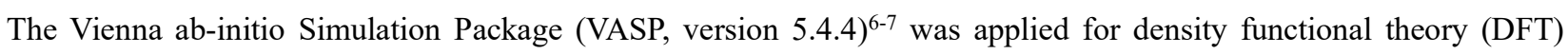
calculations. The electronic wave functions were expanded in a plane-wave basis set with a kinetic cutoff energy of $400 \mathrm{eV}$. Exchange-correlation functional was performed at Generalized gradient approximation (GGA) ${ }^{8}$ level in the form of Perdew-Burke-Ernzerhoff (PBE) $)^{9}$. The DFT $+\mathrm{U}$ method $^{10}$ was applied to describe the localized $d$ electrons of metal center in phthalocyanine rings. The effective Coulomb $(\mathrm{U})$ is $4 \mathrm{eV}$, which is widely used in similar metallic phthalocyanine based compounds. ${ }^{11-13}$ Monolayer of each COF was modeled by adding a vacuum space of $20 \AA$ in the $z$-direction to prevent the interaction between layers. Monkhorst-Pack ${ }^{14}$ Gamma-centered scheme of $k$-points sampling with $1 \times 1 \times 1$ and $1 \times 1 \times 8$ dimensions were employed for monolayer and multilayers COF respectively. As seen in Figures S1-2, the k-points are $\Gamma(0,0,0), \mathrm{X}(0.5,0,0), \mathrm{M}(0.5,0.5,0), \mathrm{Z}(0,0,0.5), \mathrm{A}(0.5,0.5,0.5)$ and $\mathrm{R}(0,0.5,0.5)$ for the band structure calculations.

\section{Effective mass calculation}

Effective mass $\left(m^{*}\right)$ calculation can be carried out through the band structures according to the following equation ${ }^{15}$ :

$$
\frac{1}{m *}=\frac{1}{\hbar} \frac{\partial^{2} E(k)}{\partial^{2} k^{2}}
$$

where, Planck's constant $\hbar=1$. $E(k)$ can be obtained from the conductive band minimum and valence band maximum according to corresponding paths of the high symmetry points.

\section{Hall effect measurements:}

The Hall effect measurement was performed on the four-point-probe Ecopia HMS-300 Hall measurement system, similar with previous reported methods. ${ }^{16}$ Hall measurement devices were fabricated by coating gold electrode on the PAE-PcM films in a square configuration (an edge length of $5 \mathrm{~mm}$; Figure S30). All measurements were conducted 
with constant magnetic field of $1.0 \mathrm{~T}$ at $300 \mathrm{~K}$. For each kind of PAE-PcM films, we have measured 3 batches to calculate the average value of conductivity, mobility, and carrier densities (as seen in Table S2).

\section{Electrochemical measurement}

The capacitance behavior was evaluated on a CHI 760E electrochemical workstation. The PAE-PcCo electrode was prepared by mixing PAE-PcCo, carbon black and PTFE in a weight ratio of 7:2:1 (trace ethanol was added to help form homogeneous suspension). The mixture was pressed into a film on carbon paper and dried under vacuum heating. The 3-electrode system were established with Pt-wire as the counter electrode and $\mathrm{Ag} / \mathrm{AgCl}$ as the reference electrode. For quasi-solid-state symmetric supercapacitors, the carbon paper supported electrode were cut into small disks with diameters of $1.13 \mathrm{~cm}$. The active material on each electrode is around $3.2 \mathrm{mg}$. Two PAE-PcCo electrodes were sandwiched with a separator and ionic electrolyte ( $0.5 \mathrm{M}$ of 1-ethyl-3-methyl-imidazolium chloride [EMIm]Cl).

As a full symmetric capacitor could be treated as two capacitors in series. The capacitance The capacitance values based on $\mathrm{CV}$ results were calculated according to the equation $(1-2)^{17}$ :

$$
\begin{aligned}
& \frac{1}{C_{\text {device }}}=\frac{1}{C_{\text {electrode }}}+\frac{1}{C_{\text {electrode }}} \\
& C_{\text {electrode }}=2 \times \frac{1}{v \times\left(V_{f}-V_{i}\right)} \int_{V_{i}}^{V_{f}} I(V) d V
\end{aligned}
$$

The $C_{\text {electrode }}$ is denoted as the capacitance contribution; $v$ is the scan rate $\left(\mathrm{V} \cdot \mathrm{s}^{-1}\right) ; V_{\mathrm{f}}$ and $V_{\mathrm{i}}$ are the integration potential

limits of the CV curve; and $I(\mathrm{~V})$ is the voltametric discharge current $(\mathrm{A}) ; \int_{V_{i}}^{V_{f}} I(V) d V$ is the integrated area from the CV curves.

Normalized capacitance to specific surface area was calculated using the following equation (3):

$$
\mathrm{C}=\frac{\mathrm{C}_{\text {electrode }}}{\mathrm{m} \times S_{B E T}}
$$

The $C\left(\mathrm{~F} \mathrm{~cm}^{-2}\right)$ refers to the normalized capacitance to specific surface area. $\mathrm{m}$ is the active material mass of each electrode. $\mathrm{S}_{\mathrm{BET}}$ is the surface area of PAE-PcCo.

\section{Capacitance contribution}

\section{Method 1.}

The capacitance contribution in PAE-PcCo based active material was firstly analyzed by the CV data at different sweep rates (equation 4$)^{18}$.

$$
i=a \times v^{b}
$$

$I$ correspond to the current and $v$ is the scan rate. Both $\mathrm{a}$ and $\mathrm{b}$ are constant parameters with $\mathrm{b}$-value determined from the slope of $\log i$ versus $\log v$. There exist two kinds of defined conditions: $b=1.0$ indicates a pure capacitive behavior of redox reactions; $b=0.5$ means a diffusion-controlled process.

\section{Method 2.}

Generally, current response at a fixed potential could be ascribed to two kinds of mechanisms: 1 . Surface capacitive process; 2. Diffusion-controlled insertion process (equation 5-6). 


$$
\begin{aligned}
& I_{(V)}=k_{1} v+k_{2} v^{0.5} \\
& I_{(V)} / v^{0.5}=k_{1} v^{0.5}+k_{2}
\end{aligned}
$$

$k_{1} v$ and $k_{2} v^{0.5}$ correspond to the current contributions from surface capacitive effects and diffusion-controlled insertion process. Therefore, we could be able to quantify the contribution of these two mechanisms at specific potentials.

\section{Method 3.}

The reciprocal of specific capacitance $\left(C^{-1}\right)$ against the square root of scan rates $\left(v^{-0.5}\right)$ could yield a linear correlation between them. Specifically, the correlation can be described by the following Equation (7).

$$
C^{-1}=R v^{0.5}+C_{T}^{-1}
$$

$C$ was experimental capacitance; $v$ was the scan rate and $C_{T}$ was the total capacitance. Notably, $C_{T}$ equals the sum of electric double layer (EDL) capacitance and pseudo-capacitance.

Plotting $C$ against the reciprocal of square root of scan rates $\left(v^{0.5}\right)$ should also give a linear correlation described by the following Equation (8)

$$
C=R v^{-0.5}+C_{d l}
$$

Extrapolating the linear fitting line to y-axis will result in the minimum $C_{d l}$. Subtraction of $C_{d l}$ from $C_{T}$ yielded the maximum Cp. 

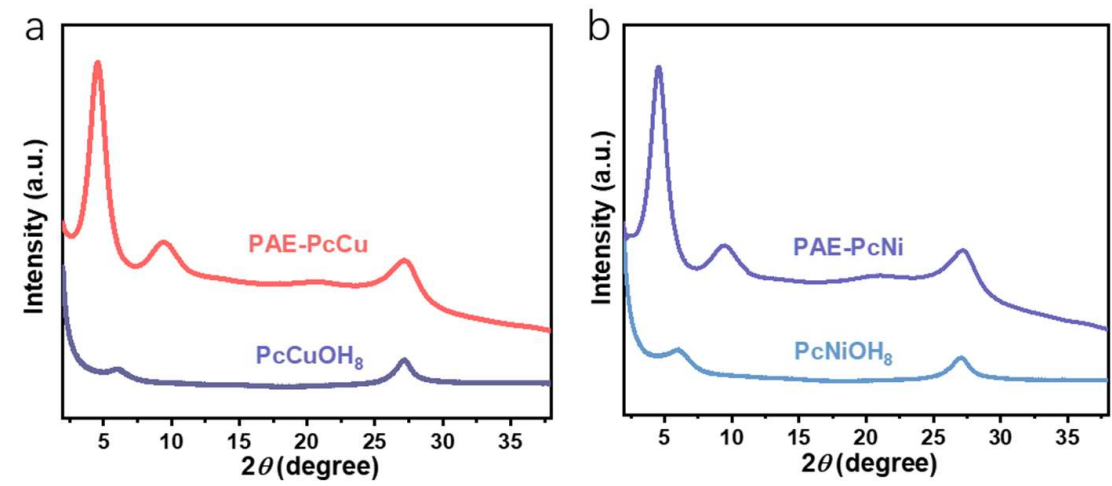

Figure S1. (a) WAXS patterns of monomer $\mathrm{PcCuOH}_{8}$ and $\mathrm{PAE}-\mathrm{PcCu}$; (b) WAXS patterns of monomer $\mathrm{PcNiOH}_{8}$ and PAE-PcNi.

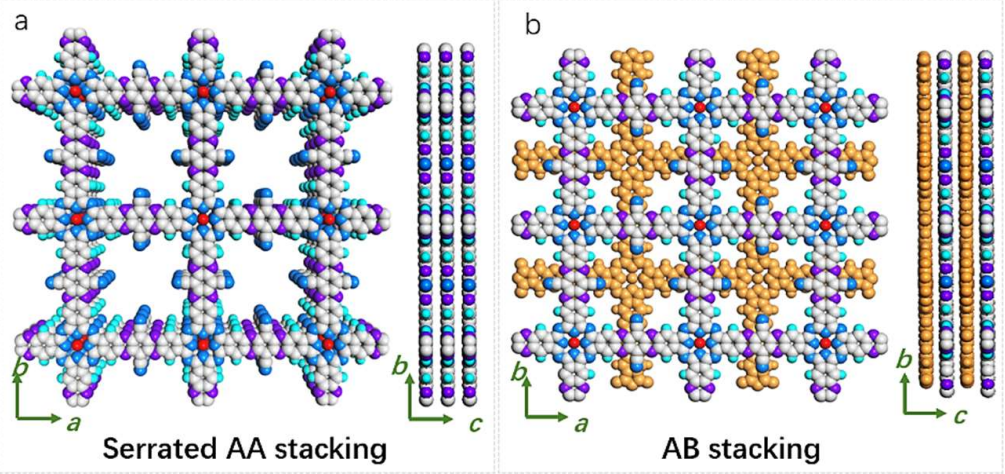

Figure S2. Top and side-view of eclipsed AA (a) and AB stacking (b) structures of PAE-PcCu.

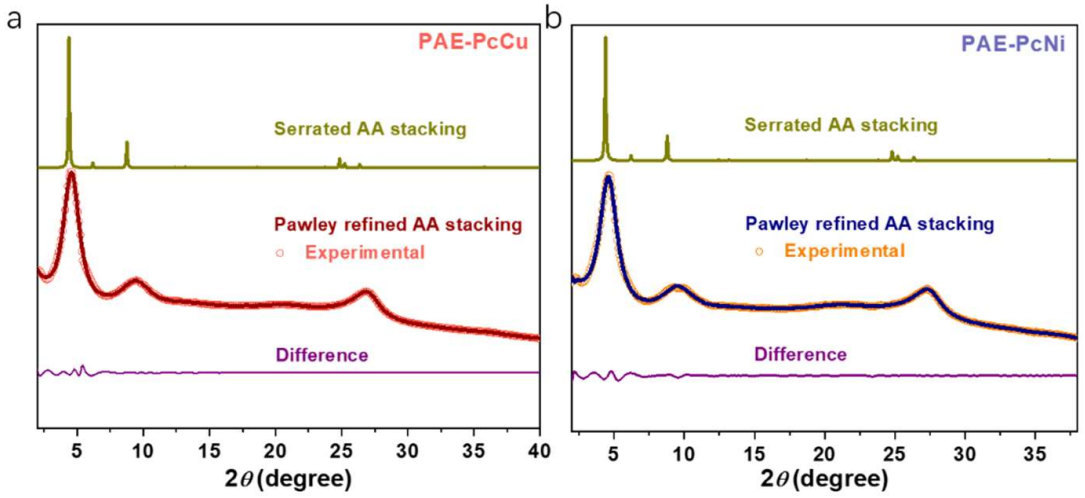

Figure S3. (a) Simulated PXRD pattern with eclipsed AA stacking geometry (light green line), experimental (red dot) and Pawley refined (red line) diffraction patterns as well as the corresponding difference plot (purple) for PAE-PcCu. The refinement result showed good agreement factors with $w R p=0.96 \%$ and $R p=0.46 \%$. (b) Simulated PXRD pattern with eclipsed AA stacking geometry (light green line), experimental (orange dot) and Pawley refined (navy line) diffraction patterns as well as the corresponding difference plot (purple) for PAE-PcNi. The refinement result showed good agreement factors with $\mathrm{wRp}=1.11 \%$ and $\mathrm{Rp}=0.71 \%$. 


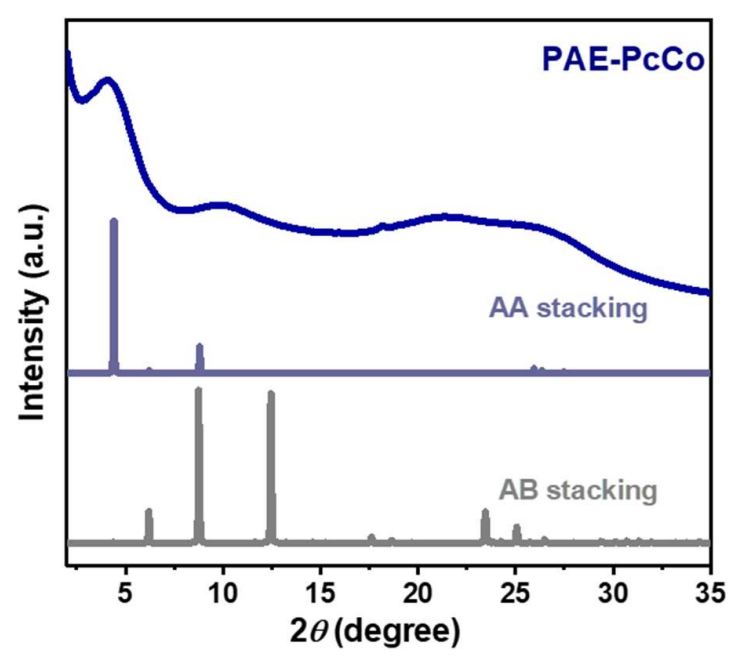

Figure S4. Experimental WAXS patterns for PAE-PcCo (navy) as well as simulated results based on eclipsed AA (light purple) and $\mathrm{AB}$ (gray) stacking manners $(\lambda=1.54 \AA)$.

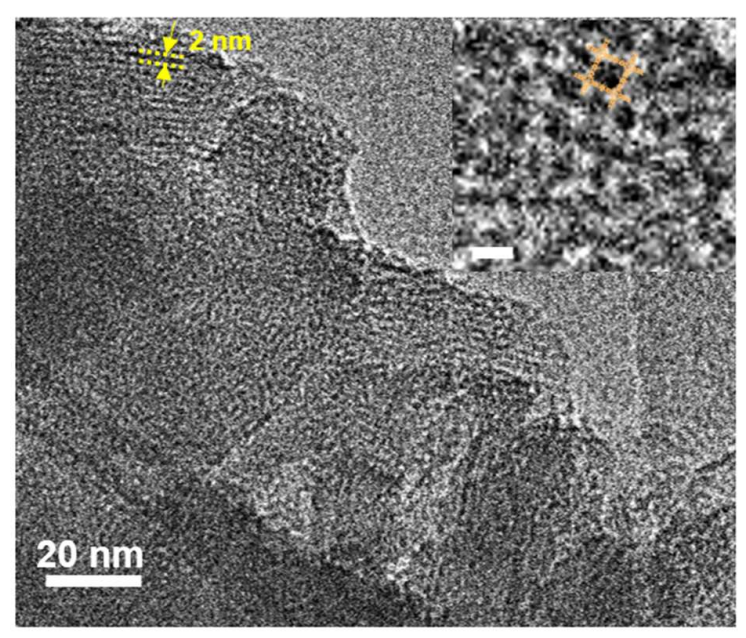

Figure S5. HRTEM image of PAE-PcNi. Insert: HRTEM image with higher magnification, indicating the square pattern with unit size of $\sim 2 \mathrm{~nm}$. Scale bar: $2 \mathrm{~nm}$. 

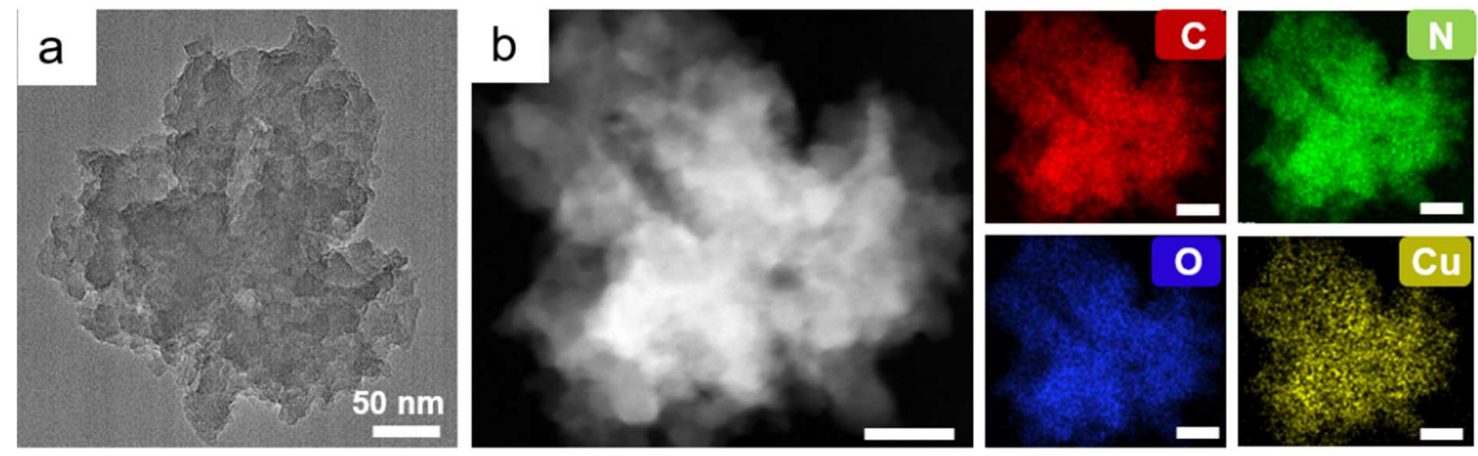

Figure S6. (a) TEM image of PAE-PcCu. (b) Dark field TEM image and its related elemental mapping for C, N, O and $\mathrm{Cu}$. These results indicated the homogenous dispersion of $\mathrm{C}, \mathrm{N}, \mathrm{O}$ and $\mathrm{Cu}$.
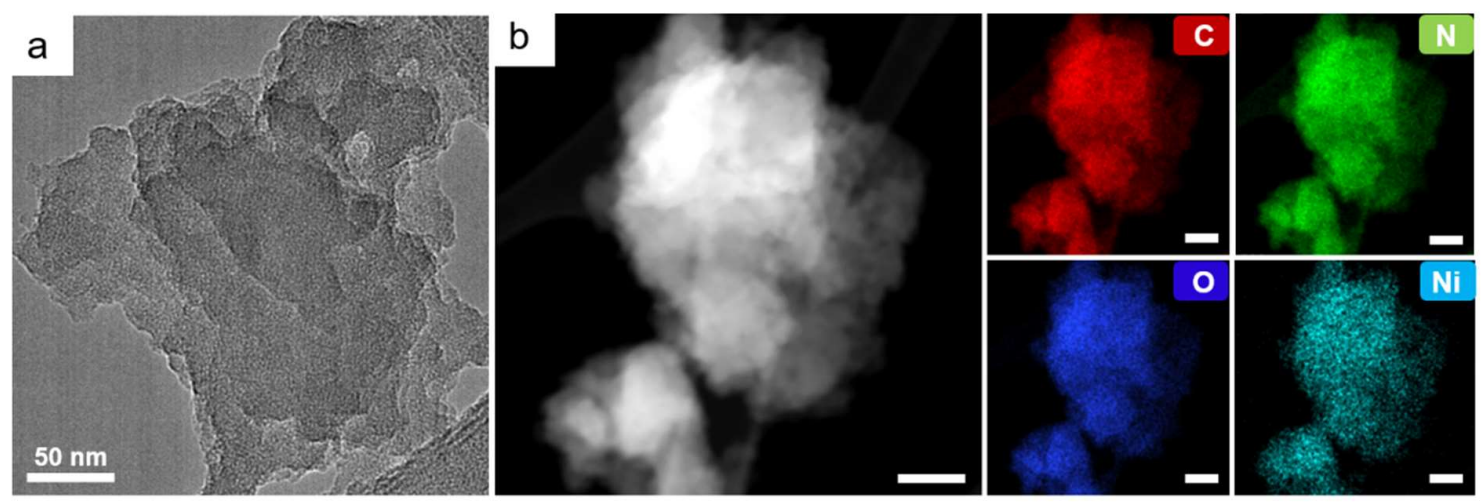

Figure S7. (a) TEM image of PAE-PcNi. (b) Dark field TEM image and its related elemental mapping for C, N, O and $\mathrm{Ni}$. These results indicated the homogenous disperser of $\mathrm{C}, \mathrm{N}, \mathrm{O}$ and $\mathrm{Ni}$.
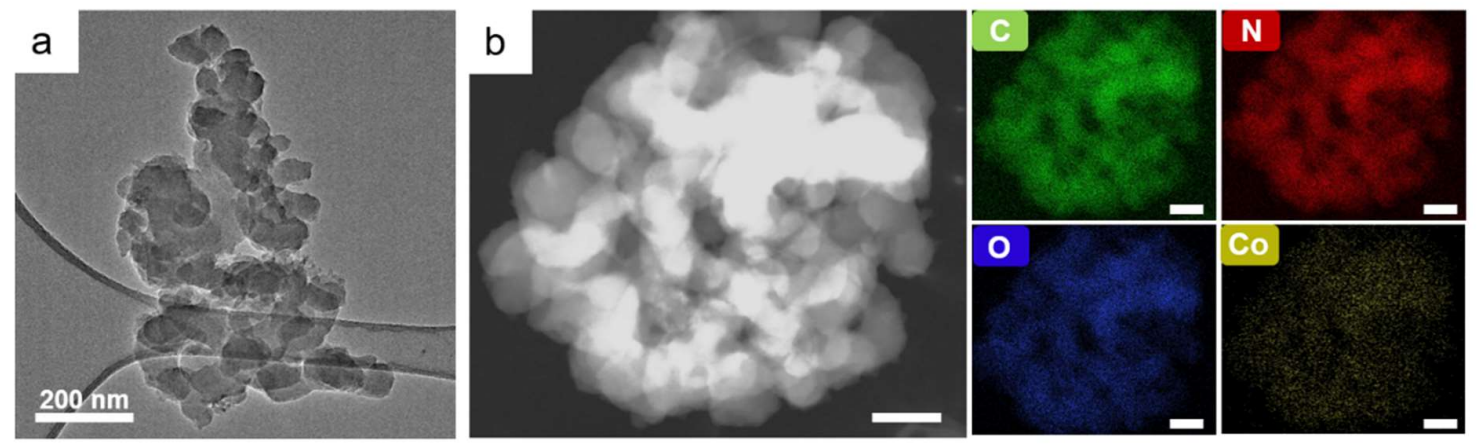

Figure S8. (a) TEM image of PAE-PcCo. (b) Dark field TEM image and its related elemental mapping for C, N, O and $\mathrm{Co}$. These results indicated the homogenous disperser of $\mathrm{C}, \mathrm{N}, \mathrm{O}$ and $\mathrm{Co}$. 

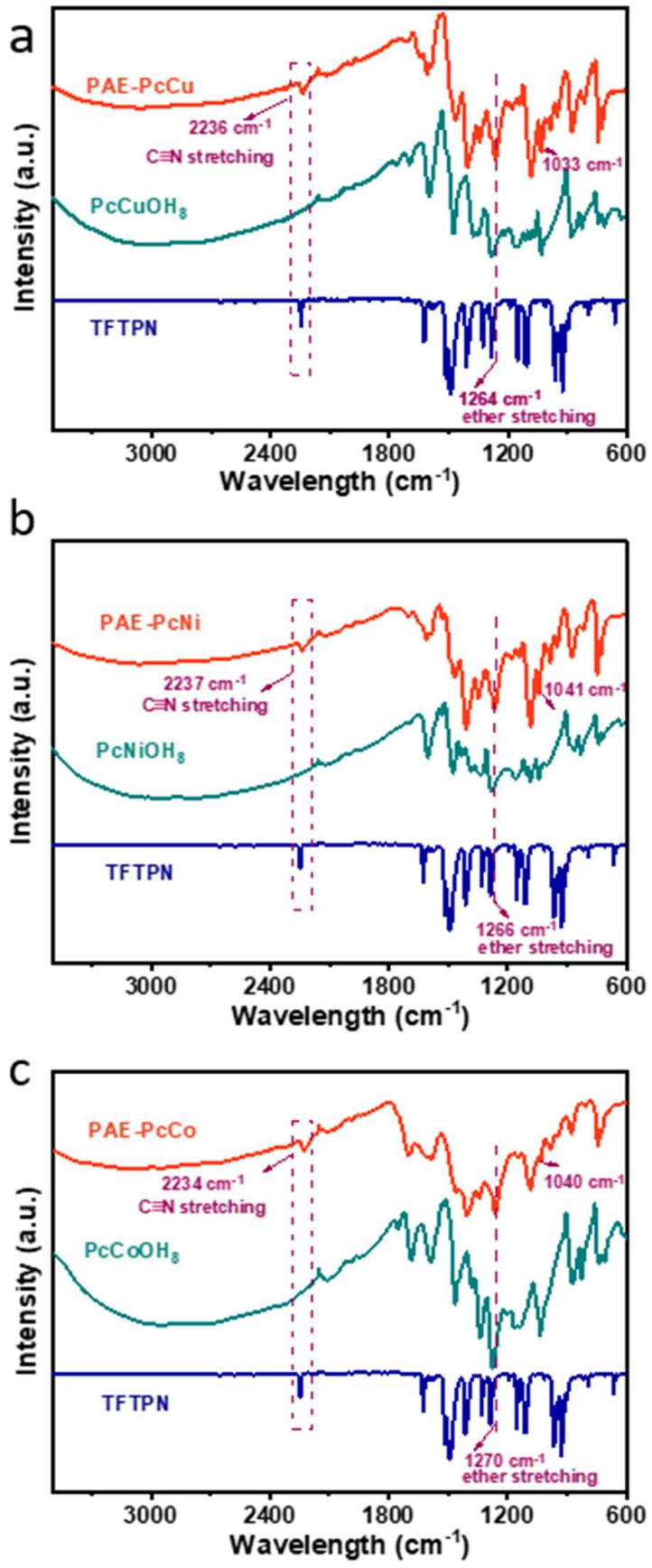

Figure S9. FTIR spectra of PAE-PcCu (a), PAE-PcNi (b) and PAE-PcCo (c). The newly appeared $\mathrm{C} \equiv \mathrm{N}$ bonds stretching in all layered COFs showed significant shift to lower wavenumbers $\left(2236 \mathrm{~cm}^{-1}, 2237 \mathrm{~cm}^{-1}\right.$ and $2234 \mathrm{~cm}^{-1}$ for PAE-PcCu, PAE-PcNi and PAE-PcCo compared with that of TFTPN monomer $\left(2247 \mathrm{~cm}^{-1}\right)$. This could be caused by the extended conjugation plane in the 2D COF frameworks. Notably, the significantly reduced broad peak at 3000$3400 \mathrm{~cm}^{-1}$ (corresponding to the $\mathrm{OH}$ stretching signals) indicated a high condensation polymerization rate. 

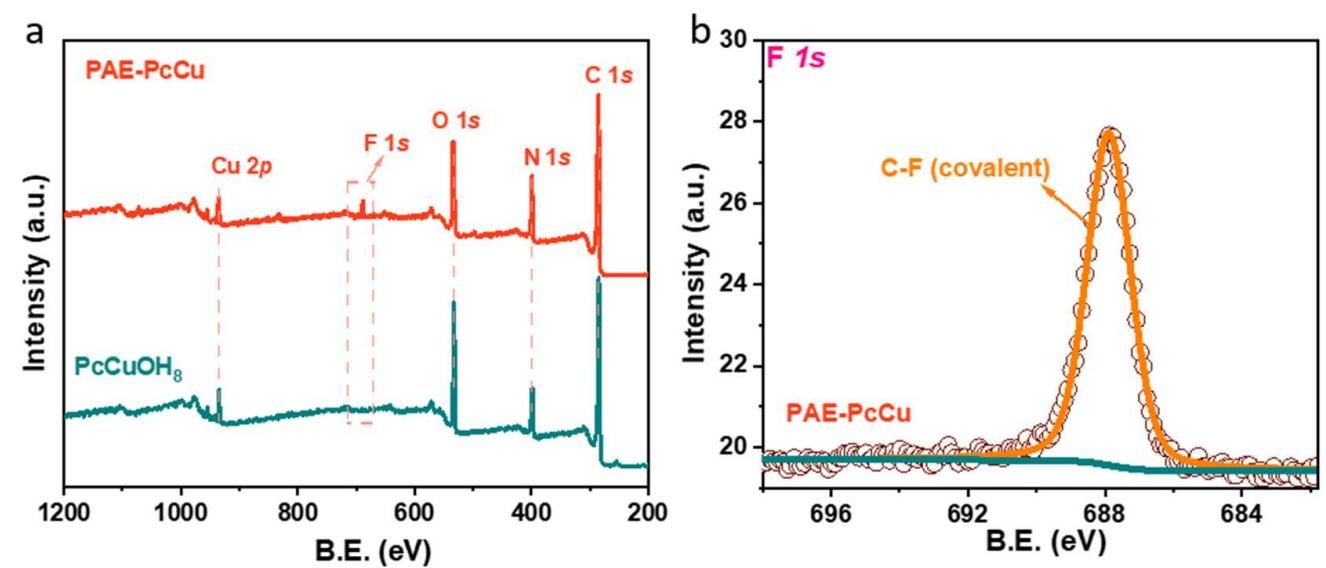

Figure S10. (a) XPS survey spectrum for PAE-PcCu and $\mathrm{PcCuOH}_{8}$. (b) High-resolution $\mathrm{F} 1 s$ core-level XPS spectra of PAE-PcCu. The residue $\mathrm{F}$ content was calculated to be $2.06 \%$, indicating a high conversion of the condensation reaction $(75.6 \%)$.
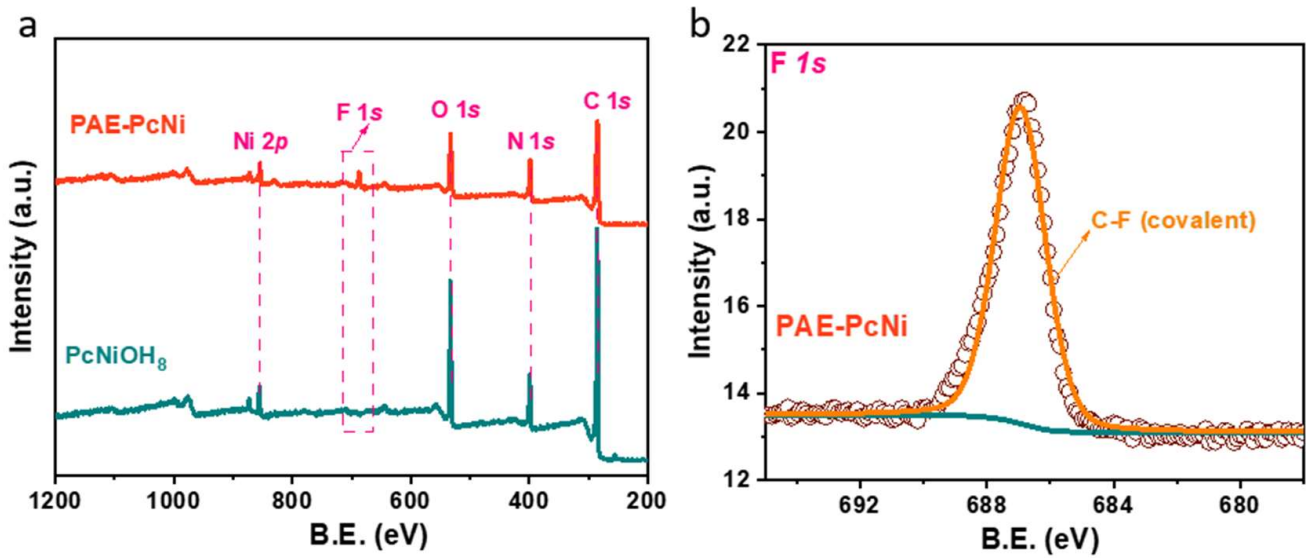

Figure S11. (a) XPS survey spectrum for PAE-PcNi and $\mathrm{PcNiOH}_{8}$. (b) High-resolution F $1 s$ core-level XPS spectra of PAE-PcNi. The content of residue $\mathrm{F}$ was $1.31 \%$ with a high condensation conversion rate of $84.5 \%$.
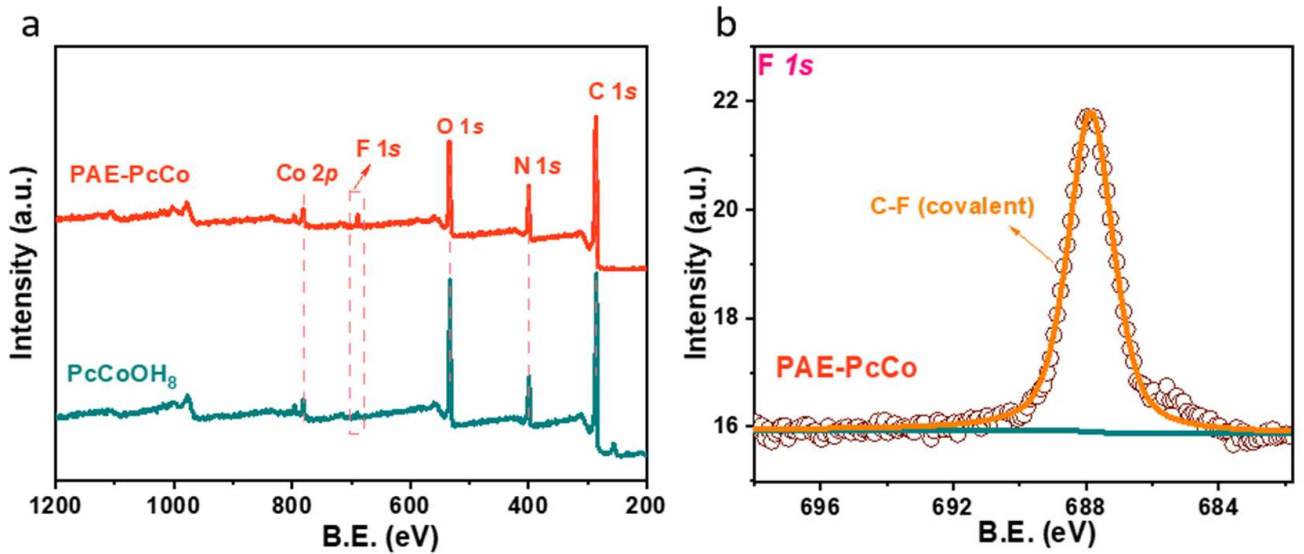

Figure S12. (a) XPS survey spectrum for PAE-PcCo and $\mathrm{PcCoOH}_{8}$. (b) High-resolution $\mathrm{F} 1 s$ core-level XPS spectra of PAE-PcCo. The residue F content was $2.12 \%$, indicating a $74.8 \%$ conversion rate. 


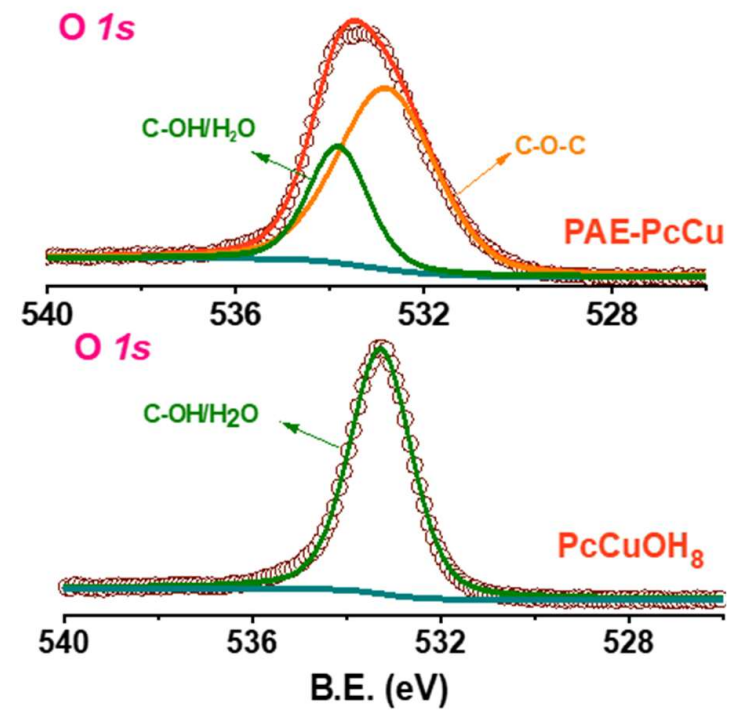

Figure S13. High-resolution O $1 s$ core-level XPS spectrum of $\mathrm{PAE}-\mathrm{PcCu}$ and $\mathrm{PcCuOH}_{8}$.

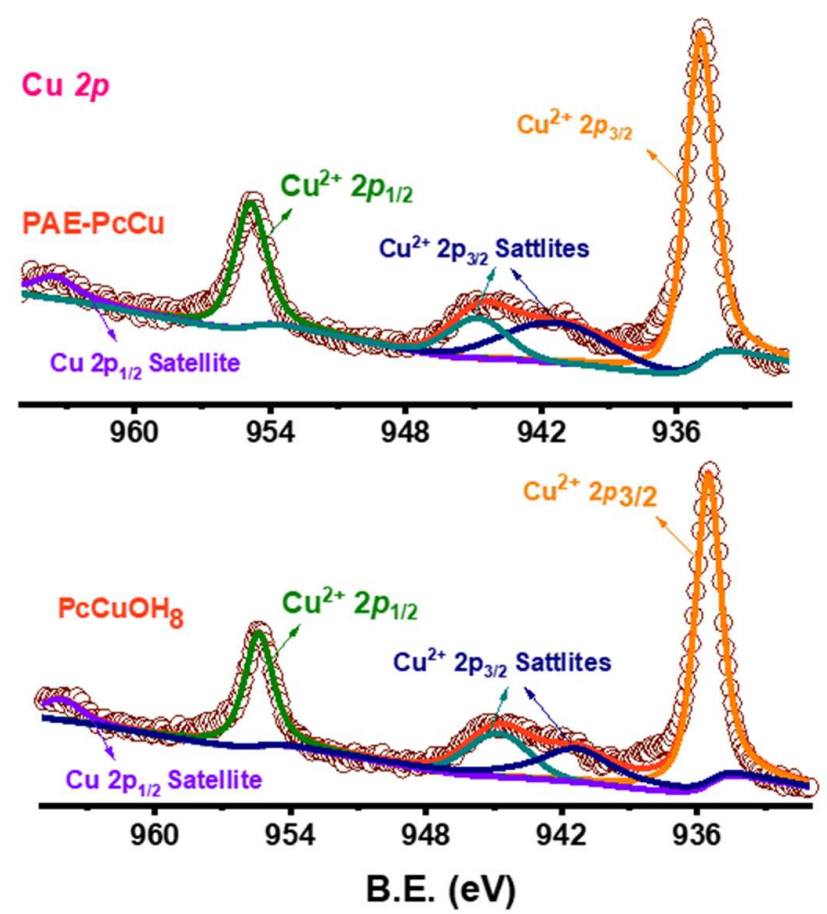

Figure S14. High-resolution $\mathrm{Cu} 2 p$ core-level XPS spectra of $\mathrm{PAE}-\mathrm{PcCu}$ and $\mathrm{PcCuOH}_{8}$. 
a

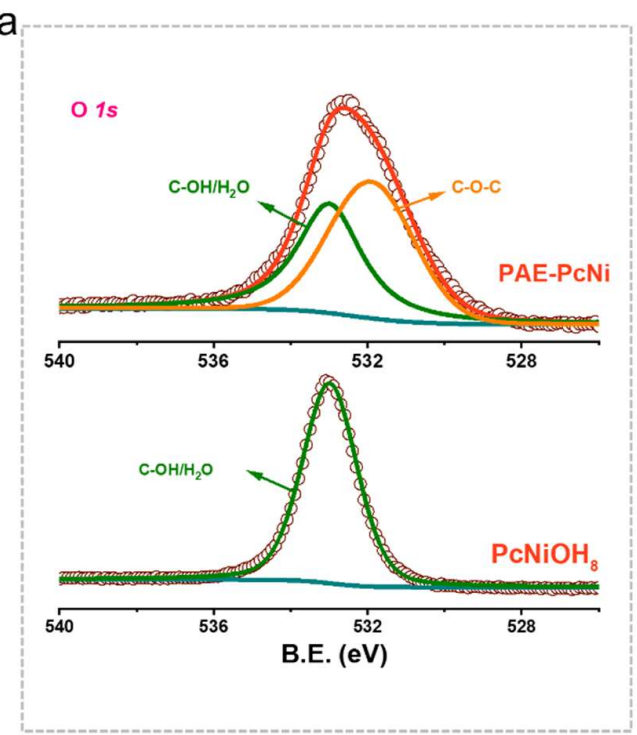

b-

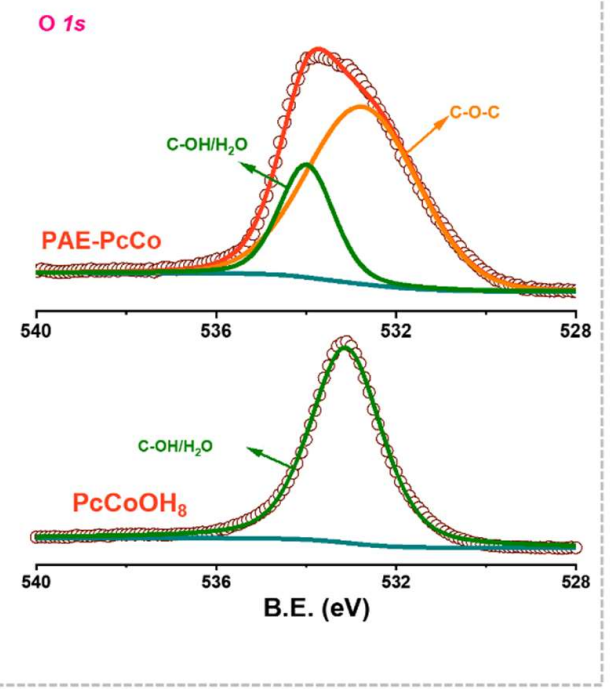

Figure S15. (a) High-resolution O $1 s$ core-level XPS spectra of PAE-PcNi and $\mathrm{PcNiOH}_{8}$. (b) High-resolution O $1 s$ core-level XPS spectra of PAE-PcCo and $\mathrm{PcCoOH}_{8}$.

a

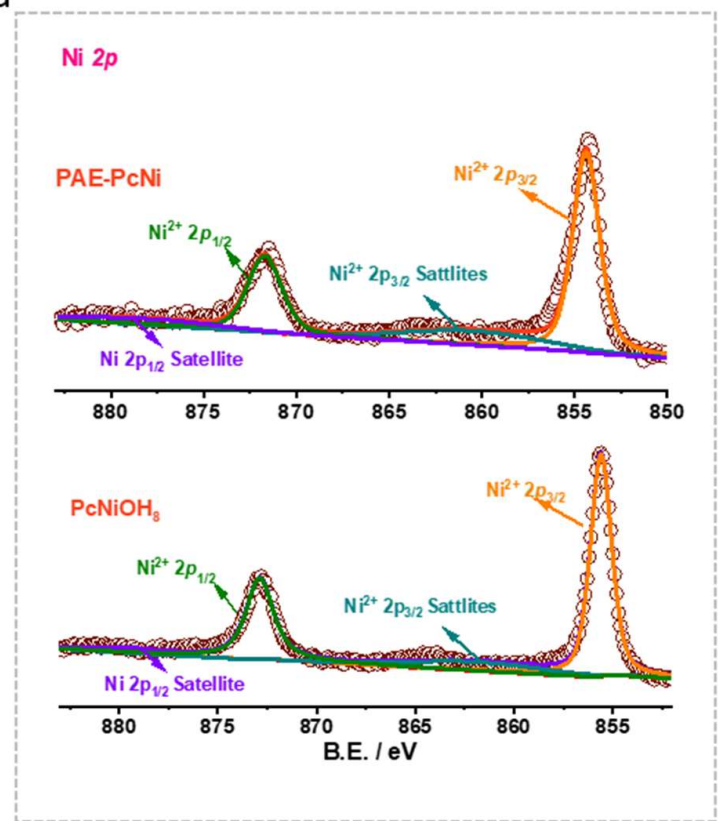

b

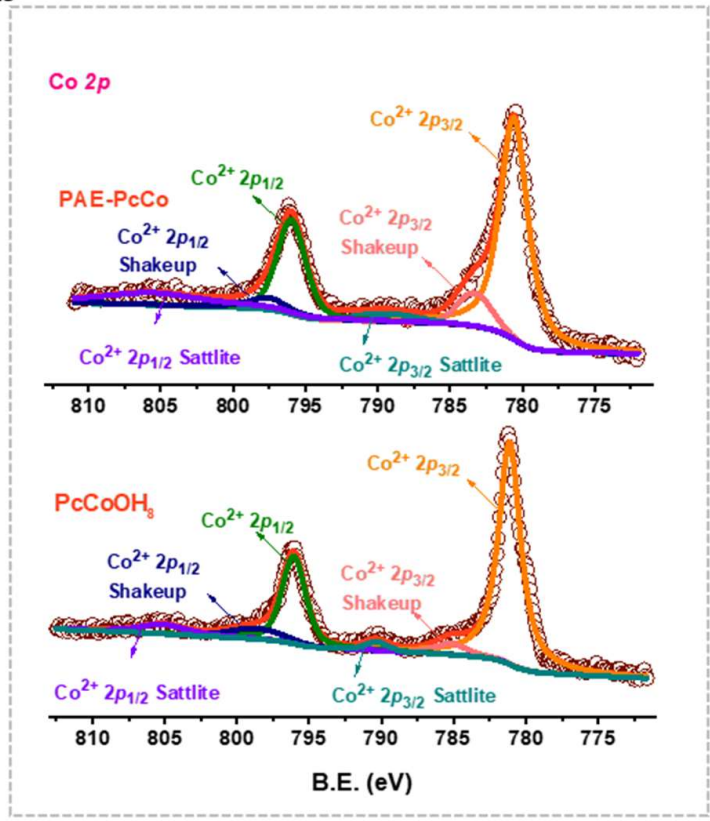

Figure S16. (a) High-resolution Ni $2 p$ core-level XPS spectra of PAE-PcNi and $\mathrm{PcNiOH}_{8}$. (b) High-resolution Co $2 p$ core-level XPS spectra of PAE-PcCo and $\mathrm{PcCoOH}_{8}$. 


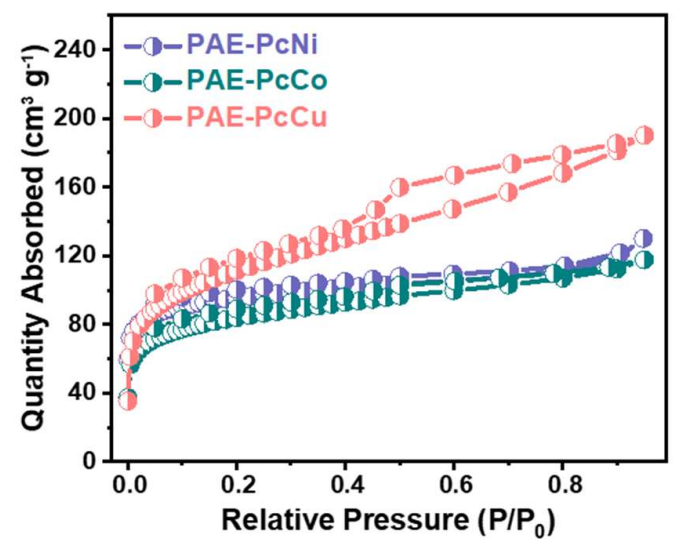

Figure S17. Nitrogen sorption isotherms for PAE-PcCu, PAE-PcNi and PAE-PcCo COFs at $77 \mathrm{~K}$.
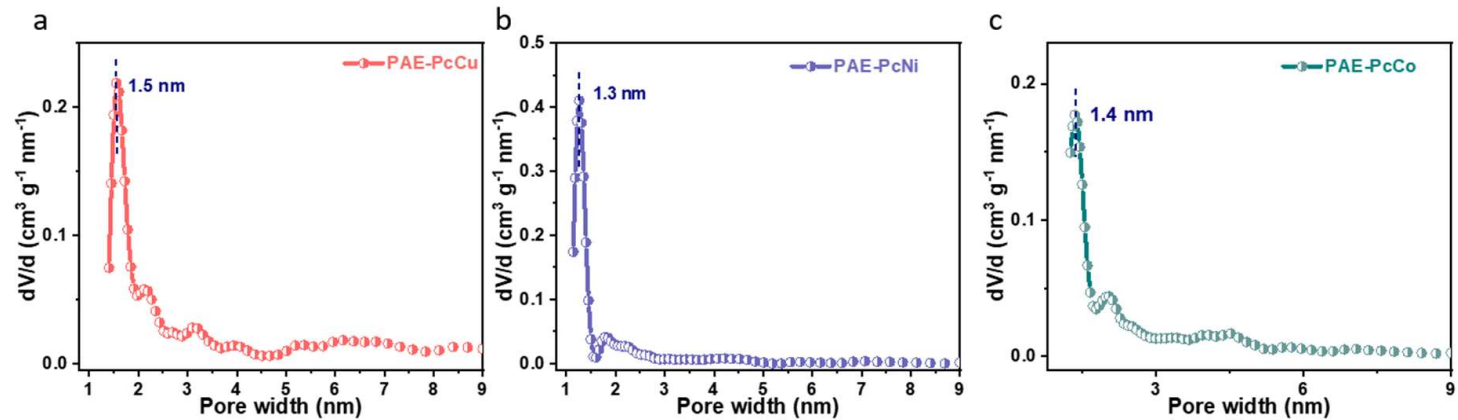

Figure S18. Pore size distribution curves for PAE-PcCu (a), PAE-PcNi (b) and PAE-PcCo (c). 


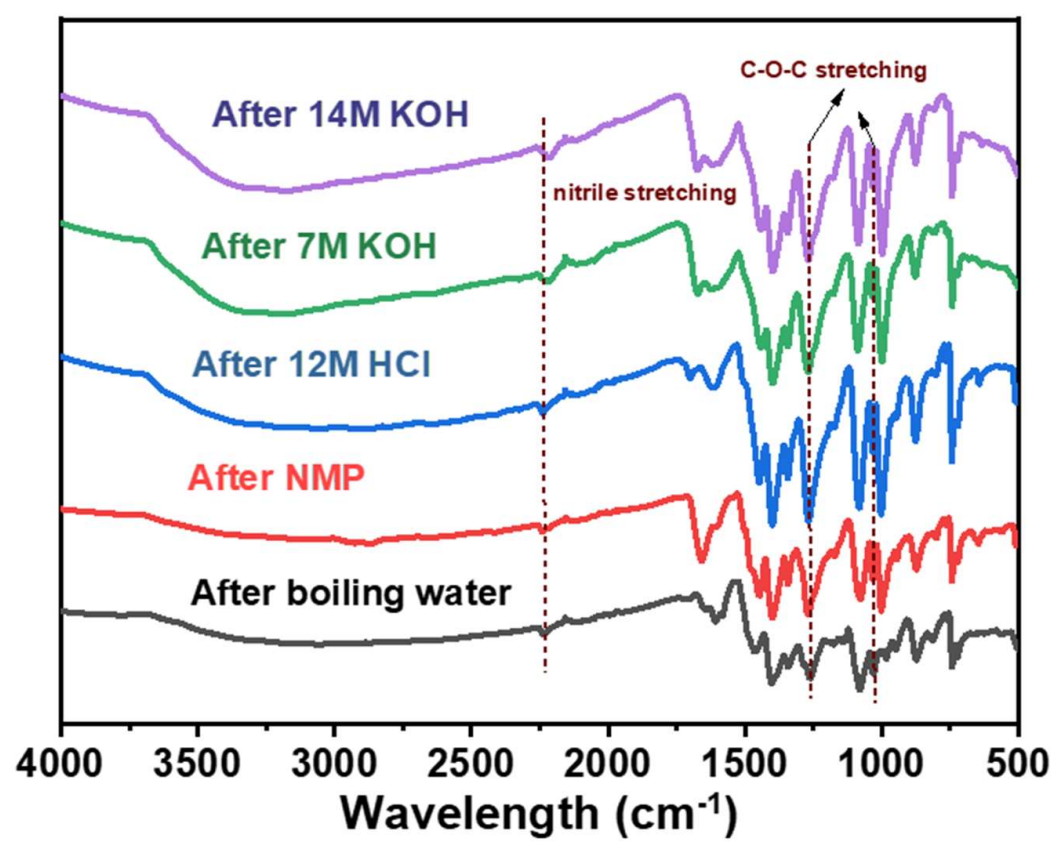

Figure S19. FT-IR patterns of PAE-PcCu after $24 \mathrm{~h}$ treatment with different conditions. The maintaining nitrile and C$\mathrm{O}-\mathrm{C}$ stretching peaks indicating the robustness of $\mathrm{PAE}-\mathrm{PcCu}$ under harsh chemical environments.

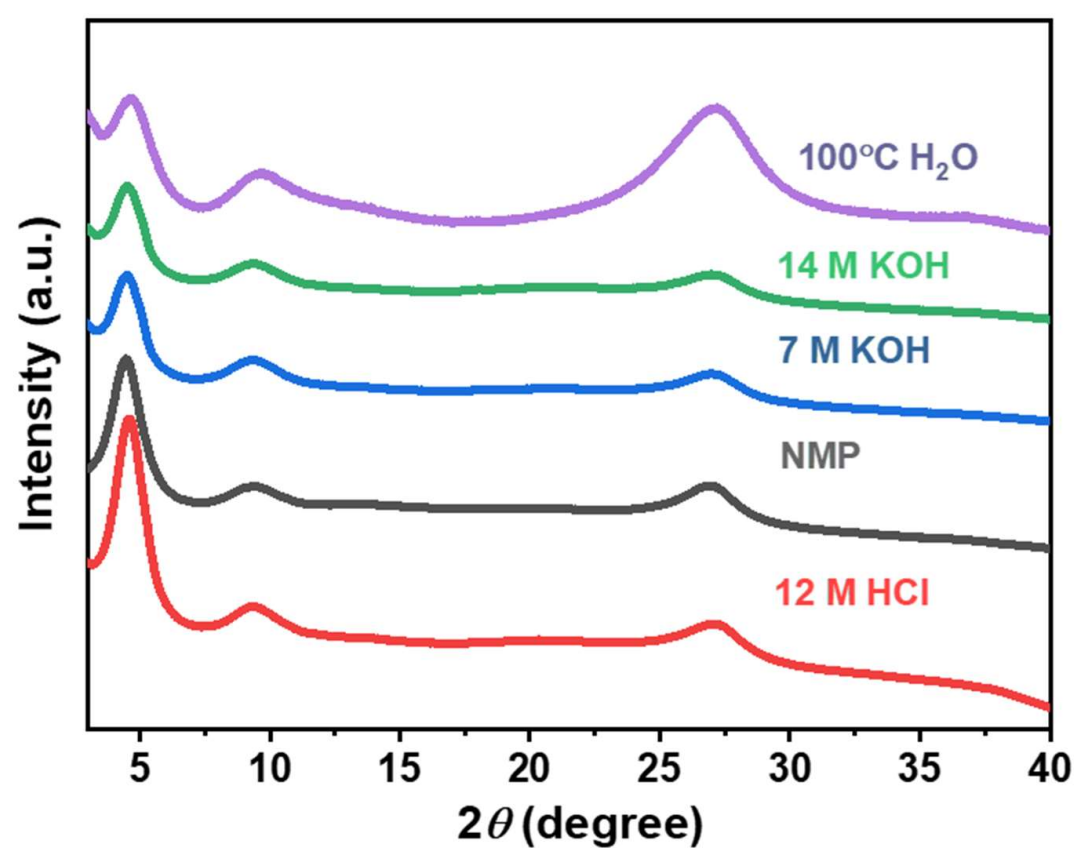

Figure S20. PXRD patterns of PAE-PcCu after $24 \mathrm{~h}$ treatment with different conditions, indicating high structural stability under harsh chemical conditions. 

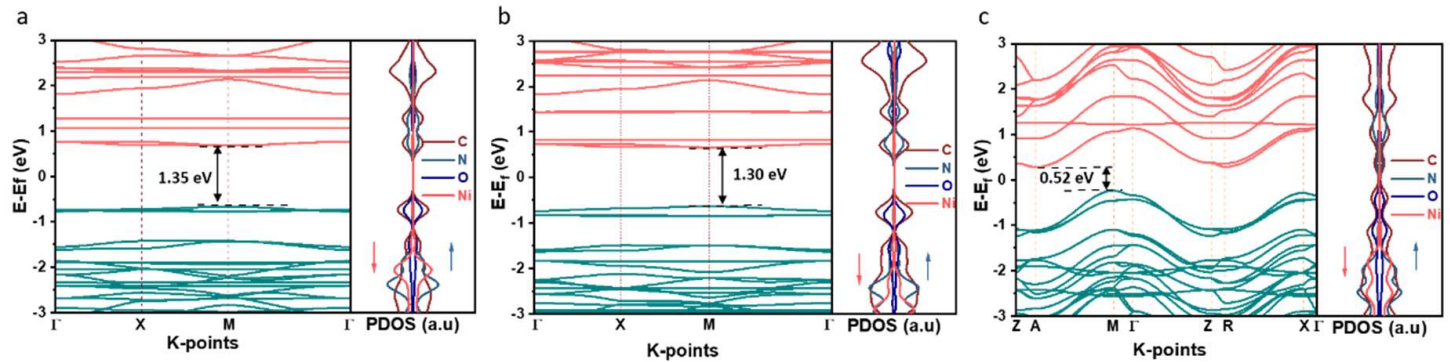

Figure S21. (a) Calculated band structure of a monolayer PAE-PcNi with GGA+U correction (left panel) and the corresponding projected density of states (PDOS) for $\mathrm{C}(p), \mathrm{N}(p), \mathrm{O}(p)$ and $\mathrm{Ni}(d)$ states. (red arrow: spin-down; blue arrow: spin-up); (b) Calculated band structure of multilayer PAE-PcNi along the in-plane direction with AA stacking mode, which plotted along the high symmetry K-points $\Gamma-\mathrm{X}$ and $\mathrm{M}-\Gamma$; (c) Calculated band structure of multilayer PAEPcNi along the high symmetry K-points Z-A, M-Г, Z-R, and X-Г.
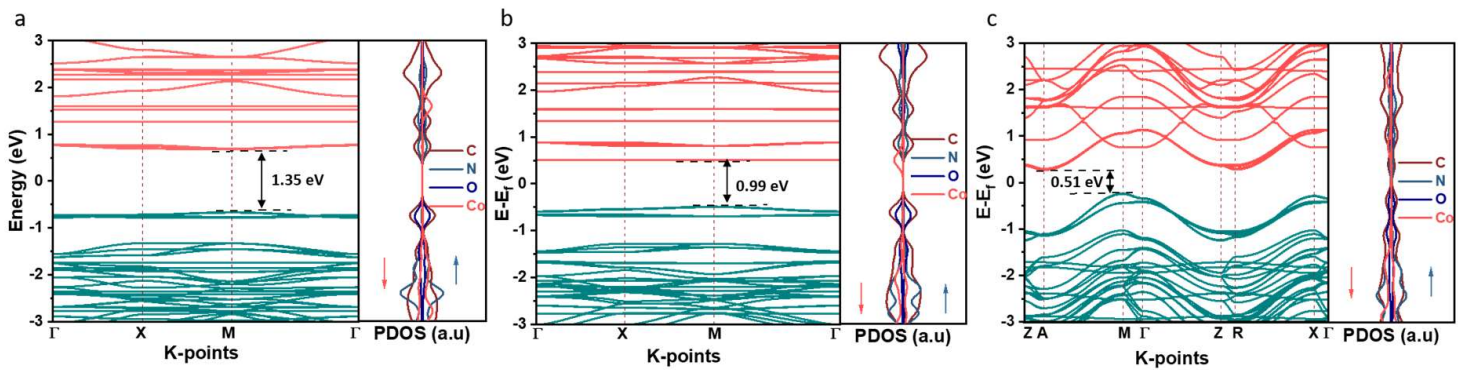

Figure S22. (a) Calculated band structure of a monolayer PAE-PcCo with GGA+U correction (left panel) and the corresponding projected density of states (PDOS) for $\mathrm{C}(p), \mathrm{N}(p), \mathrm{O}(p)$ and $\mathrm{Ni}(d)$ states. (red arrow: spin-down; blue arrow: spin-up); (b) Calculated band structure of multilayer PAE-PcCo along the in-plane direction with AA stacking mode i, which plotted along the high symmetry K-points $\Gamma-\mathrm{X}$ and $\mathrm{M}-\Gamma$; (c) Calculated band structure of multilayer PAE-PcCo along the high symmetry K-points 
a

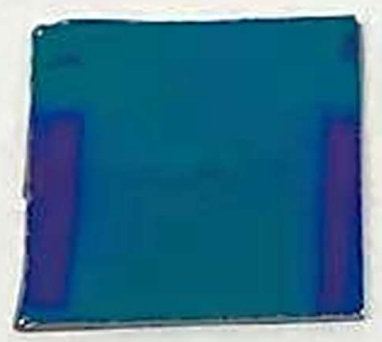

b

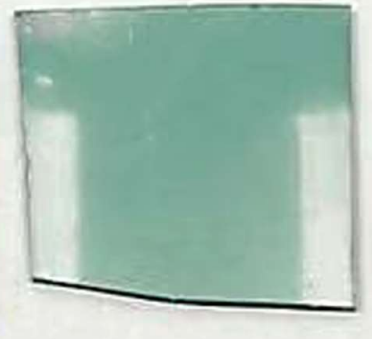

C

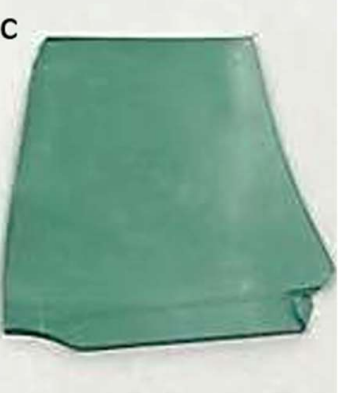

Figure S23. Images of PAE-PcCu films in-situ grown on different substrates: (a) $\mathrm{SiO}_{2} / \mathrm{Si}$; (b) ITO; (c) quartz substrates.

a

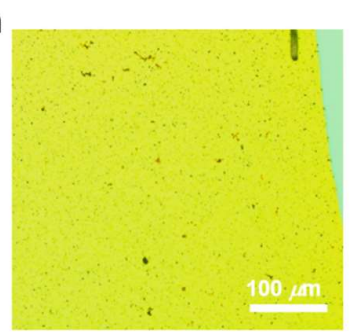

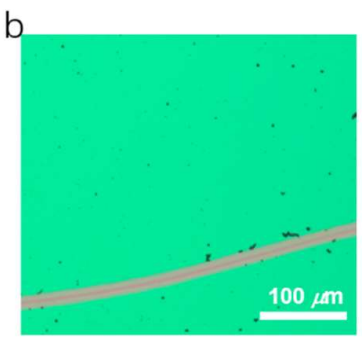

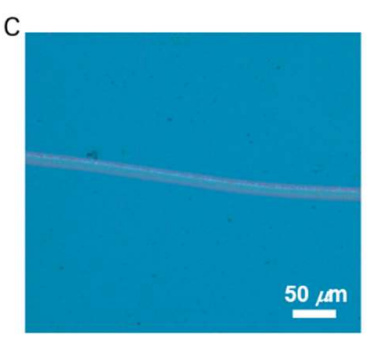

rm

Figure S24. OM images of PAE-PcCu film (a), PAE-PcNi film (b) and PAE-PcCo film (c), indicating the continuous nature of obtained PAE-PcM films.

a
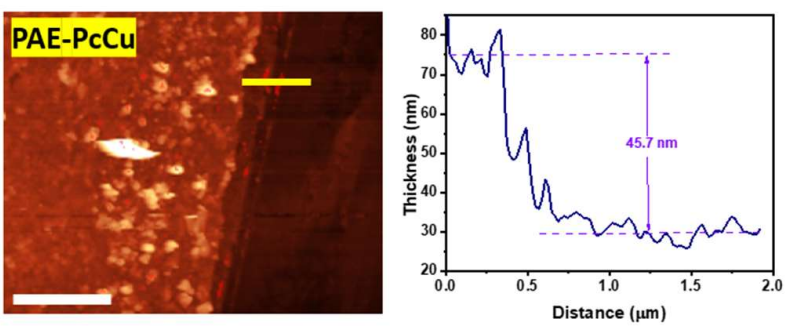

b
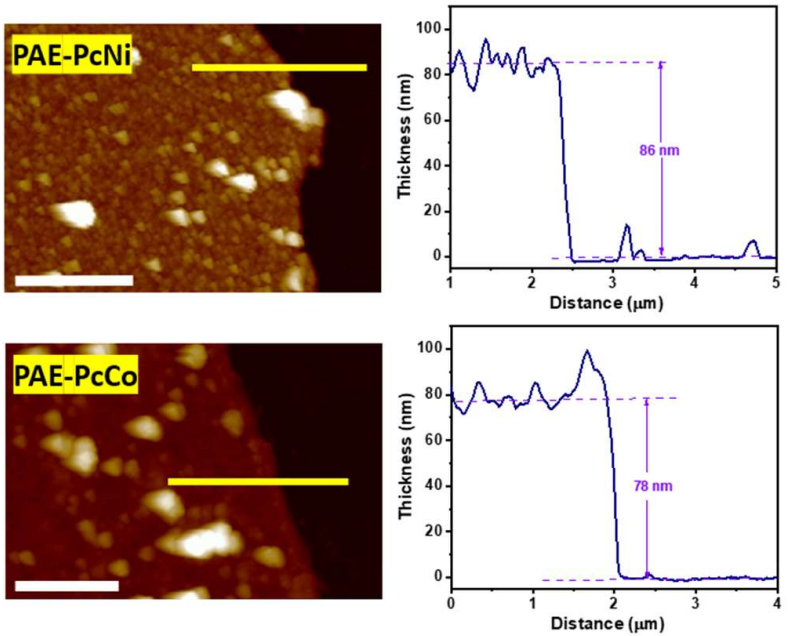

Figure S25. AFM images and corresponding height profiles for (a) PAE-PcCu film, (b) PAE-PcNi film and (c) PAEPcCo film, respectively. 

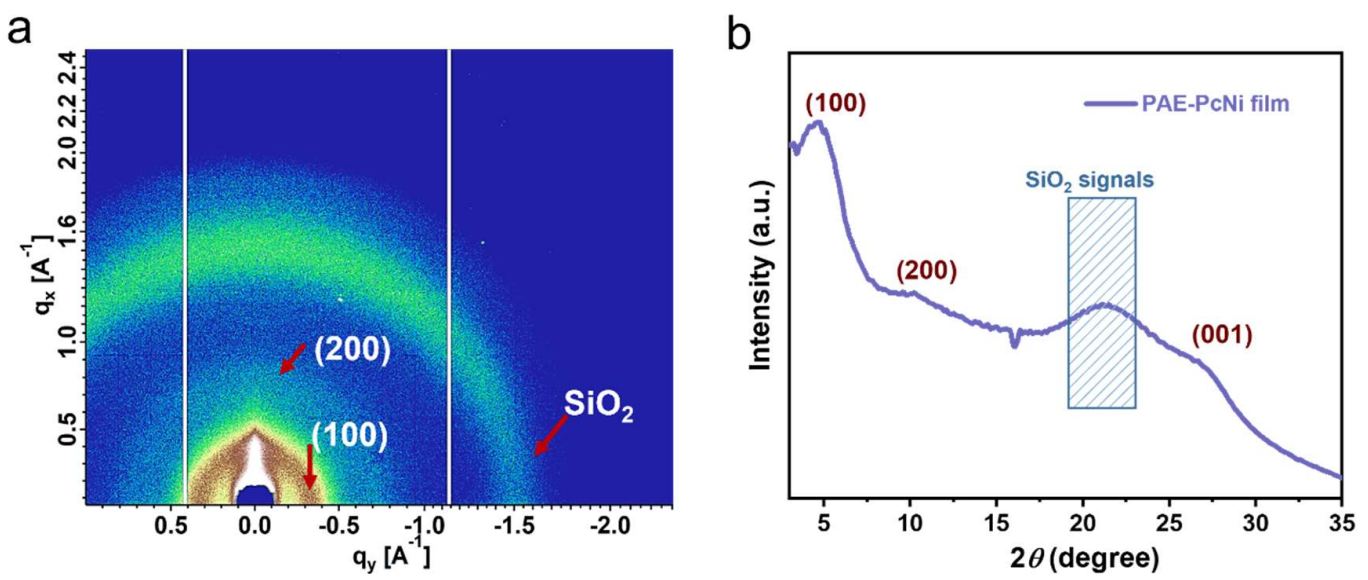

Figure S26. (a) The GIWAXS pattern of the PAE-PcNi film grown from $\mathrm{SiO}_{2} / \mathrm{Si}$ substrate; (b) $1 \mathrm{D}$ diffraction pattern converted from circular integration average of the GIWAXS result $(\lambda=1.54 \AA)$. Dotted frames indicate the $\mathrm{SiO}_{2}$ signals from the substrate. *: the artifact.
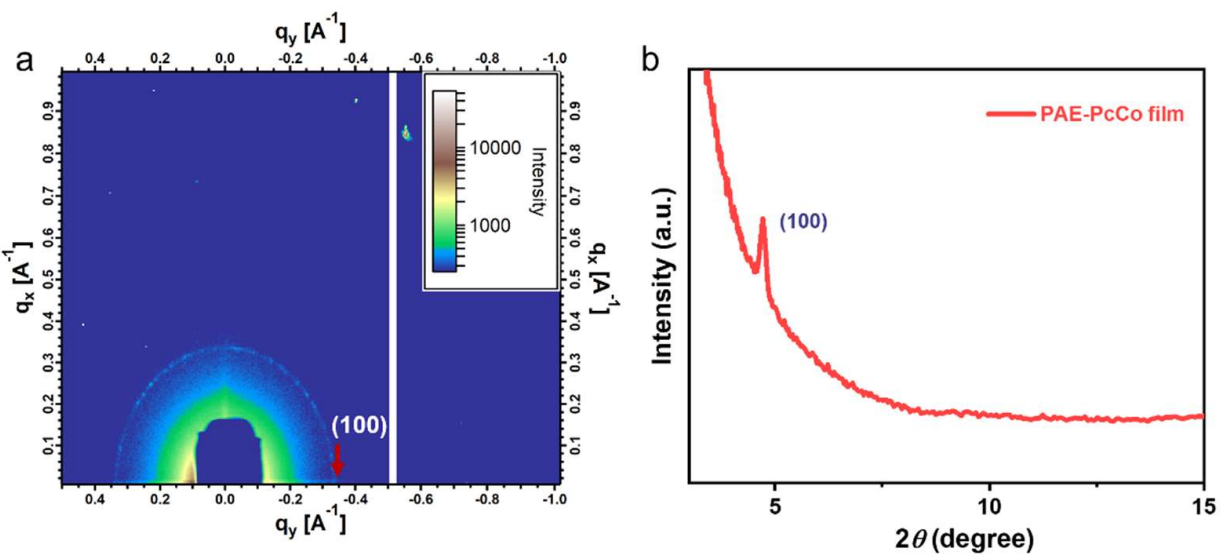

Figure S27. The GIWAXS pattern of the PAE-PcCo film grown from $\mathrm{SiO}_{2} / \mathrm{Si}$ substrate and the corresponding 1D diffraction pattern converted from circular integration average of the GIWAXS result $(\lambda=1.54 \AA)$. 


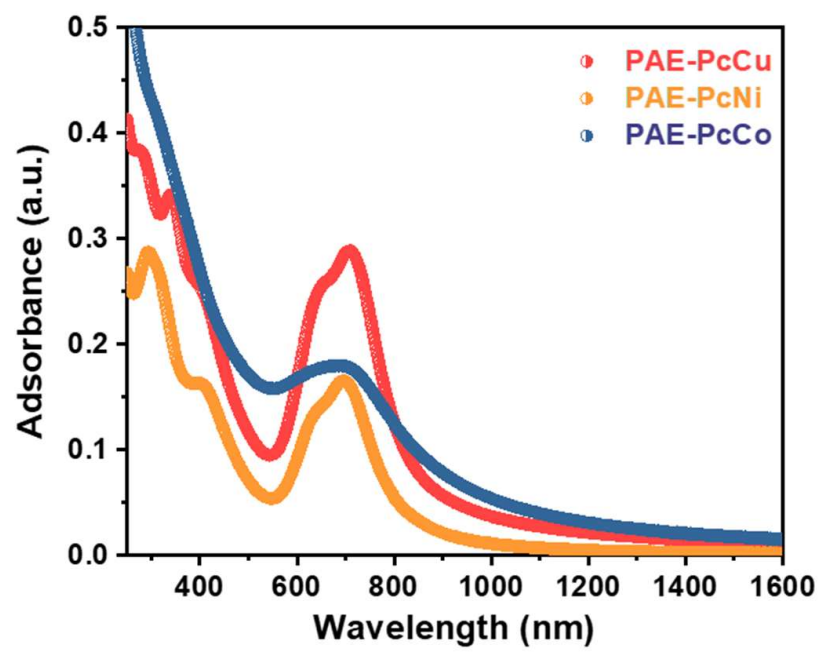

Figure S28. The UV/vis spectra for PAE-PcCu film, PAE-PcNi film and PAE-PcCo film on quartz substrates.

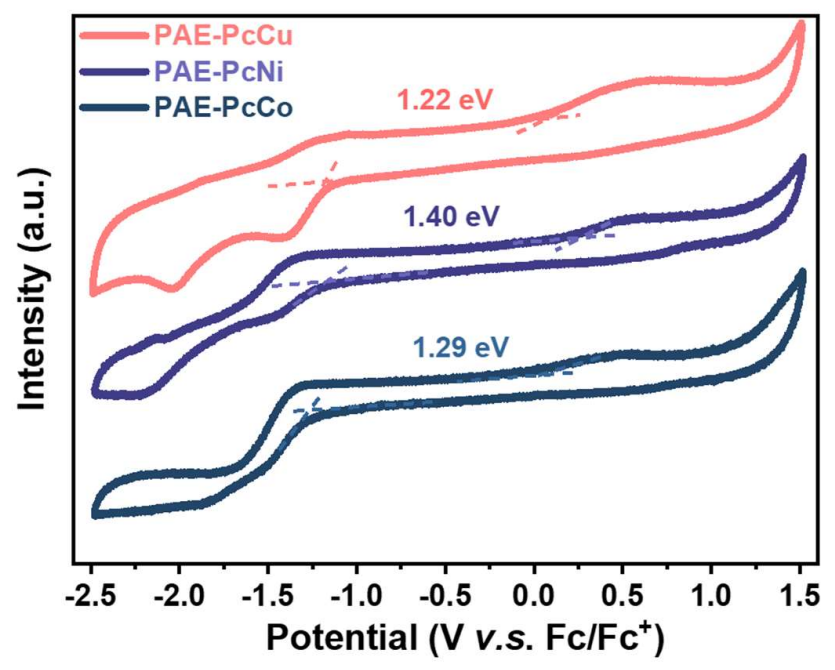

Figure S29. CV curves of PAE-PcM at the scan rate of $100 \mathrm{mV} \mathrm{s}^{-1}$. 


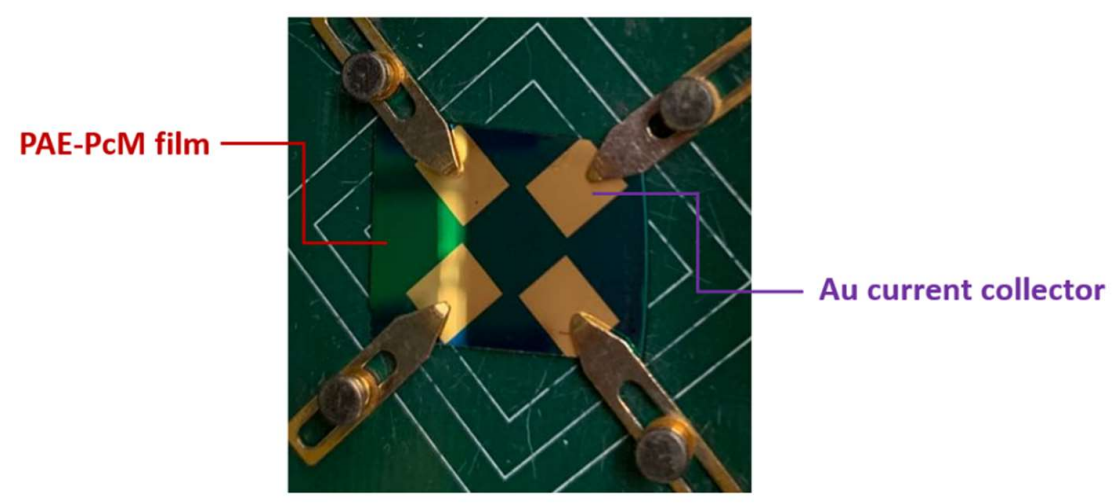

Figure S30. The photo of electrode for Hall effect measurement at magnificent field of 1T.

a

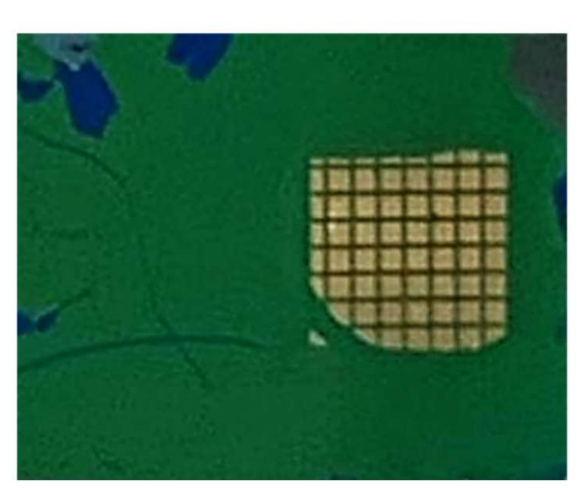

b

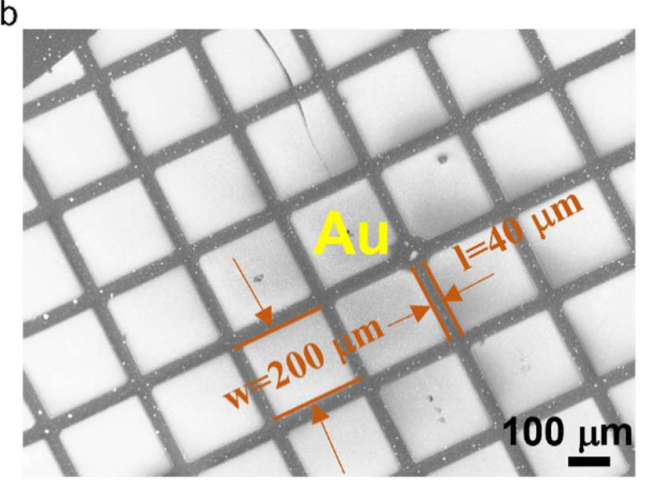

Figure S31. (a) Optical image of PAE-PcCu film after surface coated with Au electrode; (b) SEM image of square Au electrode. $l$ : distance between two gold current collectors; w: the length of gold current collector 

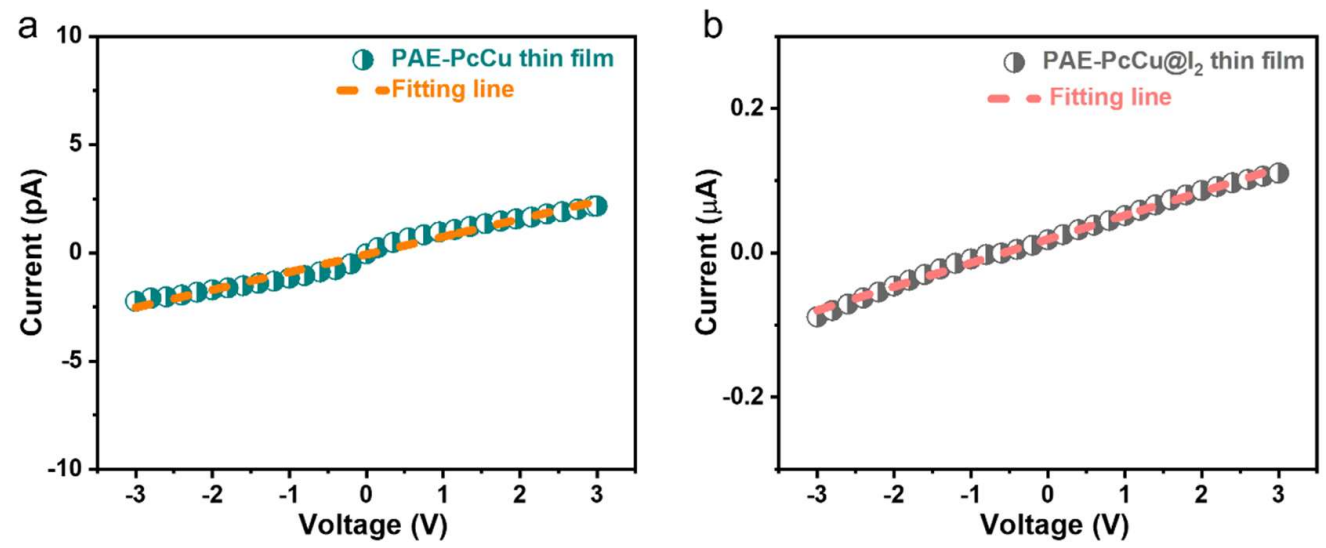

Figure S32. (a) I-V curve of PAE-PcCu film and the corresponding fitting; (b) $I-V$ curve of PAE-PcCu@ $\mathrm{I}_{2}$ thin film and the corresponding fitting. According to the equation $\sigma=k \times \frac{l}{t \times w}$ (k: slope of the fitting line; $l$ : distance between two gold current collectors; w: the length of gold current collector; t: film thickness), the conductivity for PAE-PcCu thin film is $3.80 \times 10^{-5} \mathrm{~S} \mathrm{~m}^{-1}$. Notably, the conductivity was greatly improved to $0.2 \mathrm{~S} \mathrm{~m}^{-1}$.

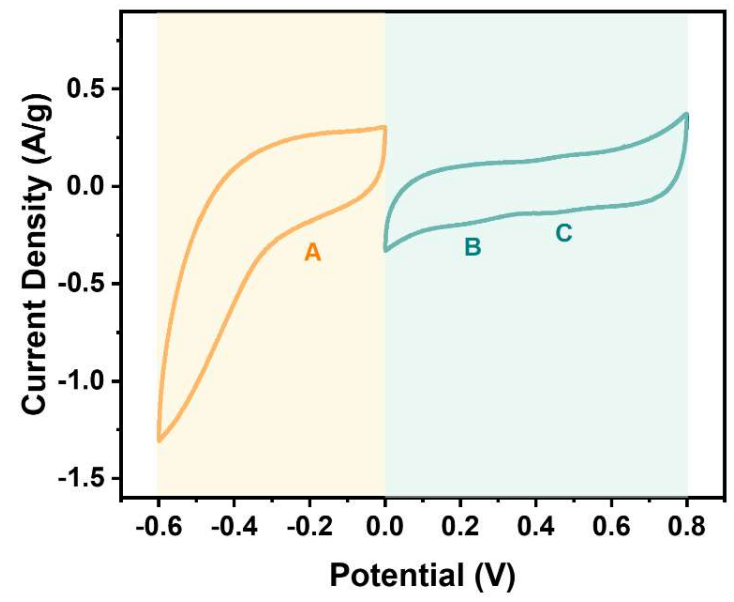

Figure S33. CV curve of PAE-PcCo in a 3-electrode system at the scan rate of $5 \mathrm{mV} \mathrm{s}^{-1}$. 


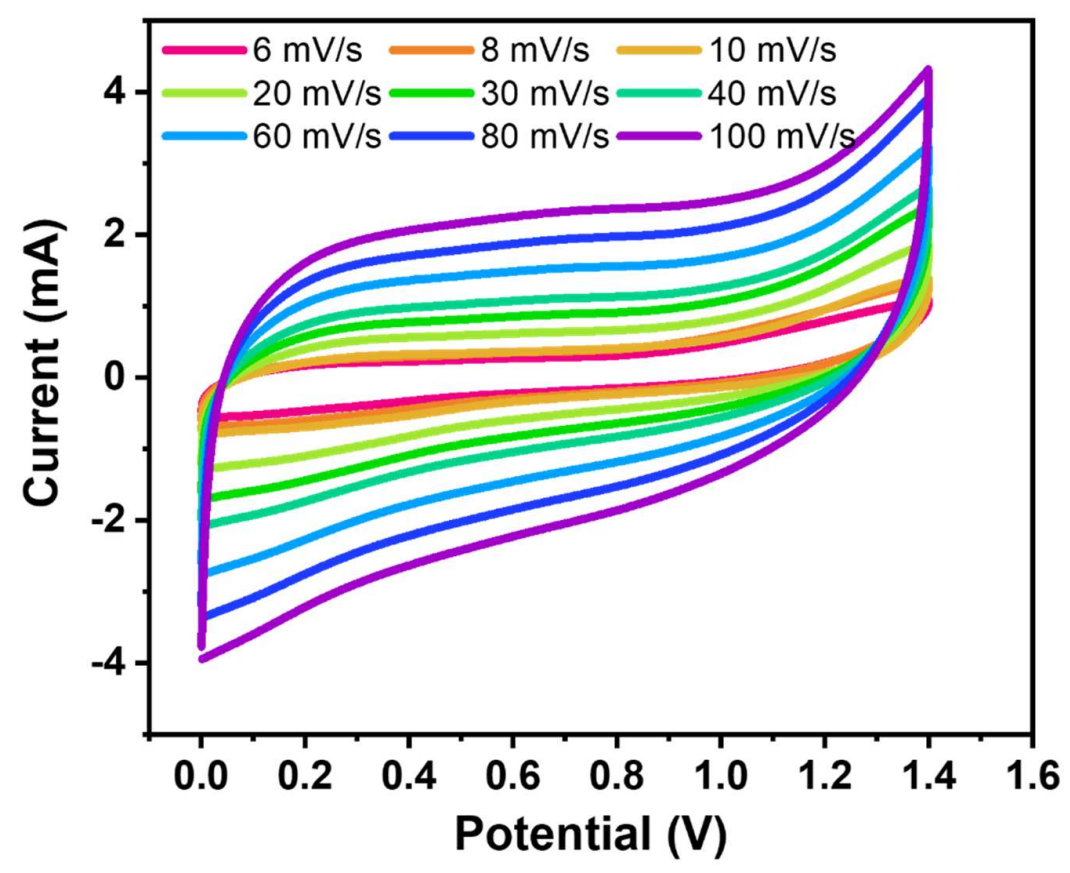

Figure S34. CV curves of PAE-PcCo based symmetric quasi-solid-state supercapacitors.
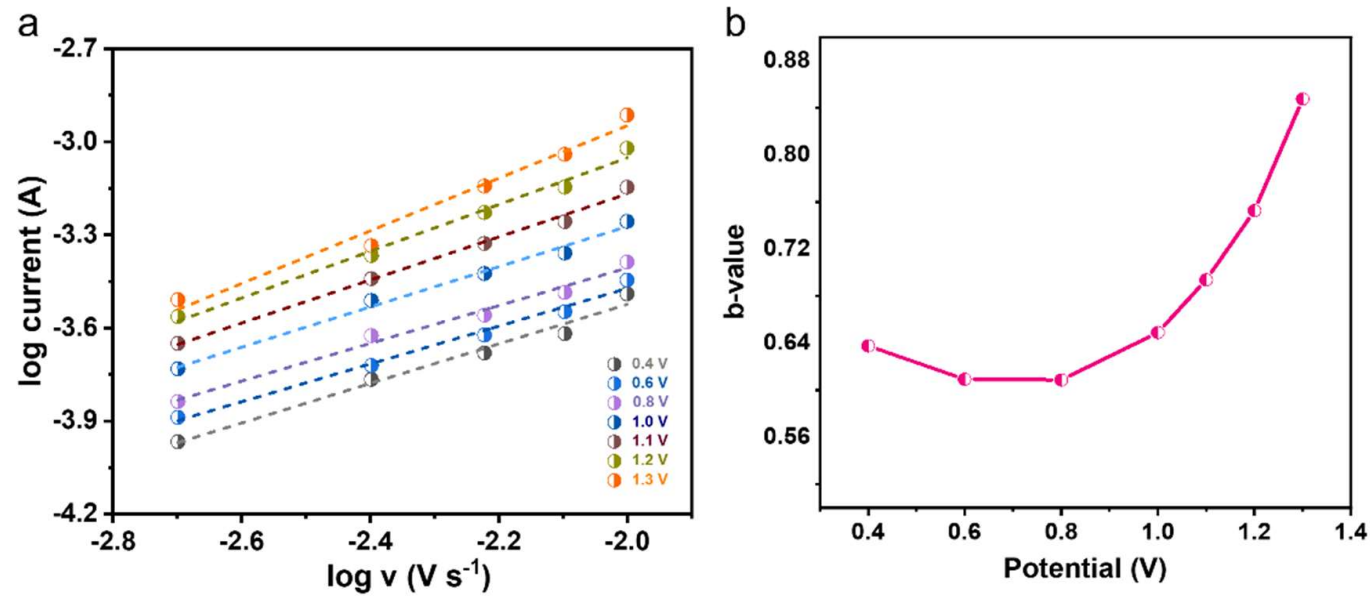

Figure S35 (a) Log current versus log v plots from different potentials in the CV curves. (b) Corresponding b values calculated from equation $i=a v^{b}$. 

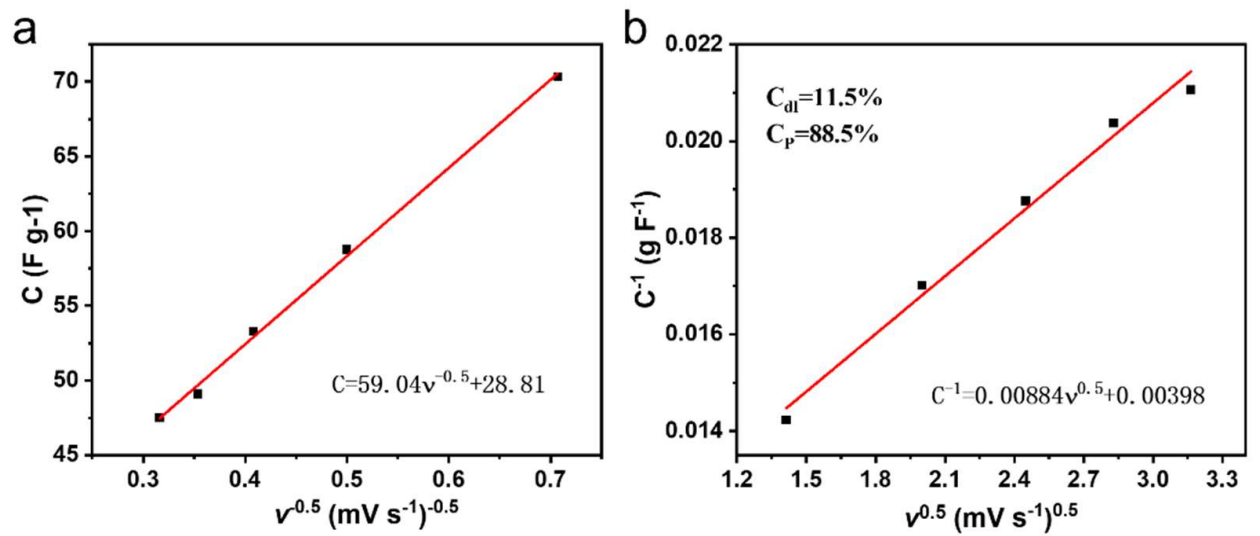

Figure S36 (a) Capacitance contribution calculation from Trasatti method: (a) Plot of reciprocal of gravimetric capacitance $(\mathrm{C})$ against square root of scan rate $\left(v^{0.5}\right)$. (b) Plot of $\mathrm{C}$ against reciprocal of square root of scan rate $\left(v^{-0.5}\right)$. Inset: Histogram showing the respective capacitance contributions.

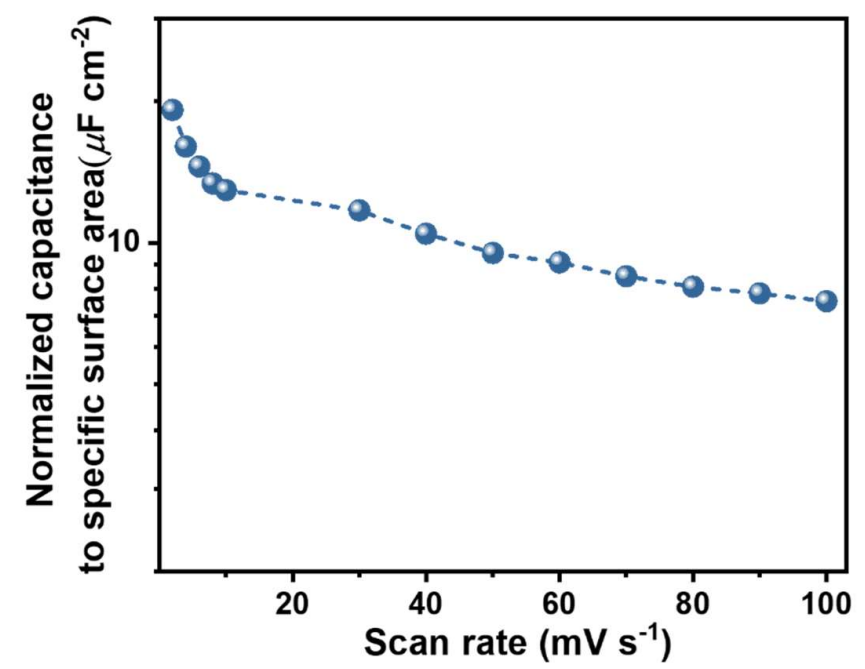

Figure S37 Normalized capacitance to specific surface area calculated from CV curves. The active material loading on each electrode is around $3.2 \mathrm{mg}$. 


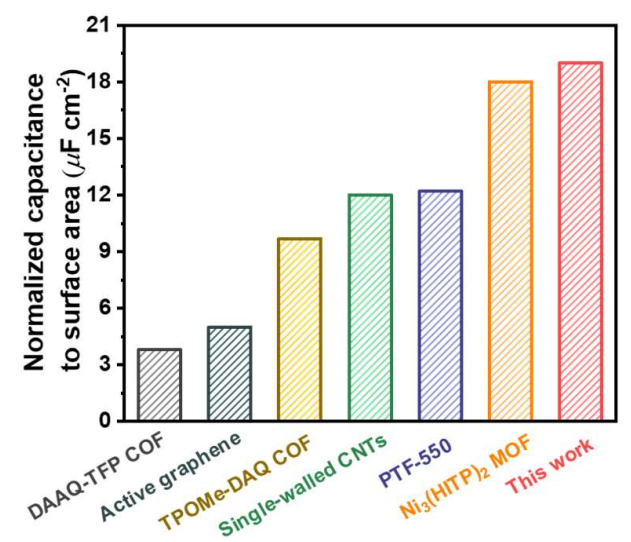

Figure S38 Comparison of normalized capacitance to specific surface area to state-of-art porous materials (DAAQTFP COF $;{ }^{20}$ Active graphene; ${ }^{21}$ TPOMe-DAQ COF; ${ }^{22}$ Single-walled CNTs; ${ }^{23}$ PTF-550; $;{ }^{24} \mathrm{Ni}_{3}(\mathrm{HITP})_{2} \mathrm{MOF} .{ }^{25}$

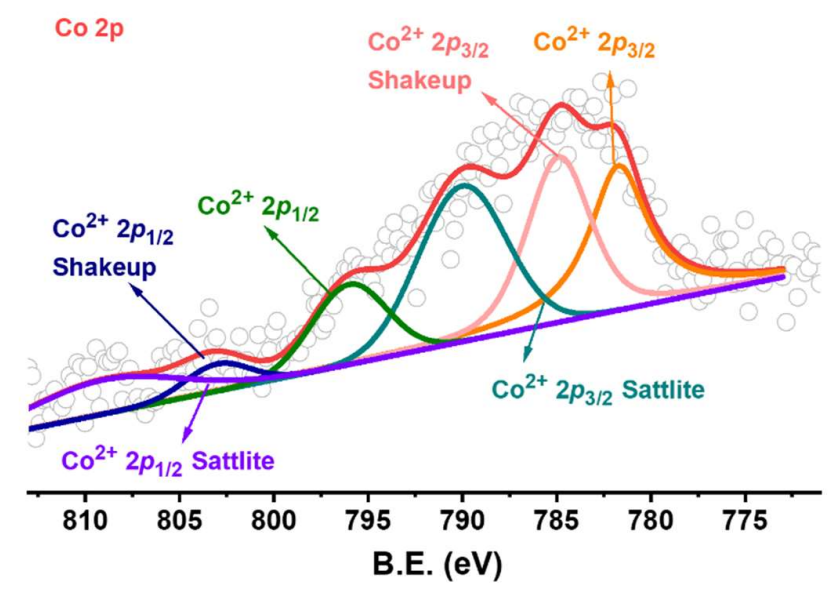

Figure S39. Core-level Co $2 p$ spectra of PAE-PcCo after long-stability test. High resolution core-level XPS spectra of Co $2 p$ showed that the Co-N peak at 781.7 and $796.0 \mathrm{eV}$ could be maintained. Additionally, no typical signals from metallic cobalt $(778.1 \mathrm{eV}), \mathrm{Co}_{3} \mathrm{O}_{4}(779.6 \mathrm{eV}), \mathrm{CoO}(780.0 \mathrm{eV})$ and $\mathrm{CoOOH}(780.1 \mathrm{eV})$ could be observed, ${ }^{19}$ indicating the good stability after long-term cycling. 
Table S1. Nitrogen physisorption properties of PAE-PcM frameworks

\begin{tabular}{ccccc}
\hline Sample & $\left.\mathbf{S}_{\text {BET }^{\mathbf{a}}} \mathbf{( m}^{\mathbf{2}} \mathbf{g}^{\mathbf{- 1}}\right)$ & $\mathbf{S}_{\text {Lang }} \mathbf{~}^{\left(\mathbf{m}^{\mathbf{2}} \mathbf{g}^{\mathbf{- 1}}\right)}$ & $\mathbf{S}_{\mathbf{m i c r o}} \mathbf{c}\left(\mathbf{m}^{\mathbf{2}} \mathbf{g}^{-\mathbf{1}}\right)$ & $\mathbf{V}_{\text {Micro }^{\mathbf{a}}}\left(\mathbf{c m}^{\mathbf{3}} \mathbf{g}^{\mathbf{- 1}}\right)$ \\
\hline PAE-PcCu & 402 & 521 & 245 & 0.112 \\
\hline PAE-PcNi & 363 & 398 & 326 & 0.136 \\
\hline PAE-PcCo & 308 & 367 & 246 & 0.108
\end{tabular}

The calculated surface areas based on a) BET model and b) Langmuir model; c) Microporous surface area; d) Microporous volume calculated at $\mathrm{P} / \mathrm{P}_{0}=0.99$.

Table S2. Carrier mobility parameters from Hall effect measurements.

\begin{tabular}{|c|c|c|c|c|}
\hline Sample & $\begin{array}{l}\text { Hall resistance } \\
\qquad(\Omega \mathrm{cm})\end{array}$ & $\begin{array}{l}\text { Conductivity } \\
\qquad\left(\mathrm{S} \mathrm{cm}^{-1}\right)\end{array}$ & $\begin{array}{c}\text { Carrier density } \\
\qquad\left(\mathrm{cm}^{-3}\right)\end{array}$ & $\begin{array}{l}\text { Carrier mobility } \\
\qquad\left(\mathrm{cm}^{-2} \mathrm{~V} \mathrm{~s}^{-1}\right)\end{array}$ \\
\hline \multirow{3}{*}{ PAE-PcCu Film } & $2.1 \times 10^{6}$ & $4.8 \times 10^{-7}$ & $8.3 \times 10^{12}$ & $3.6 \times 10^{-1}$ \\
\hline & $3.8 \times 10^{6}$ & $2.7 \times 10^{-7}$ & $5.2 \times 10^{12}$ & $3.2 \times 10^{-1}$ \\
\hline & $4.0 \times 10^{7}$ & $2.5 \times 10^{-8}$ & $7.3 \times 10^{11}$ & $2.2 \times 10^{-1}$ \\
\hline \multirow[t]{2}{*}{ Average value } & - & $2.6( \pm 2.3) \times 10^{-7}$ & $4.7( \pm 3.8) \times 10^{12}$ & $3.0( \pm 0.7) \times 10^{-1}$ \\
\hline & $9.8 \times 10^{5}$ & $1.0 \times 10^{-6}$ & $4.0 \times 10^{12}$ & 1.6 \\
\hline \multirow[t]{2}{*}{ PAE-PcNi Film } & $1.0 \times 10^{6}$ & $9.1 \times 10^{-7}$ & $2.2 \times 10^{12}$ & 2.6 \\
\hline & $1.1 \times 10^{6}$ & $8.8 \times 10^{-7}$ & $1.0 \times 10^{12}$ & 5.4 \\
\hline \multirow[t]{2}{*}{ Average value } & - & $9.3( \pm 0.6) \times 10^{-7}$ & $2.4( \pm 1.5) \times 10^{12}$ & $3.2( \pm 1.9)$ \\
\hline & $4.1 \times 10^{5}$ & $2.4 \times 10^{-6}$ & $1.1 \times 10^{12}$ & 13.6 \\
\hline \multirow[t]{2}{*}{ PAE-PcCo Film } & $6.1 \times 10^{5}$ & $1.6 \times 10^{-6}$ & $4.5 \times 10^{11}$ & 22.8 \\
\hline & $6.2 \times 10^{5}$ & $1.6 \times 10^{-6}$ & $4.6 \times 10^{11}$ & 21.9 \\
\hline Average value & - & $1.9( \pm 0.5) \times 10^{-6}$ & $0.7( \pm 0.4) \times 10^{12}$ & $19.4( \pm 5.1)$ \\
\hline
\end{tabular}


Table S3. Comparison tables of carrier mobilities of crystalline frameworks at room temperature

\begin{tabular}{cccc} 
Frameworks & Methods & $\begin{array}{c}\text { Carrier mobilities } \\
\left(\mathbf{c m}^{\mathbf{2}} \mathbf{V}^{\mathbf{- 1}} \mathbf{s}^{\mathbf{- 1}}\right)\end{array}$ & Reference \\
\hline PAE-PcCu & Hall & $0.3 \pm 0.07$ & This work \\
\hline PAE-PcNi & Hall & $3.2 \pm 1.90$ & This work \\
\hline PAE-PcCo & Hall & $19.4 \pm 5.10$ & This work \\
\hline CuPc-pz COF & Hall & $0.9 \pm 0.2$ & J. Am. Chem. Soc. 2019, 141, 16810 \\
\hline NiPc-CoTAA & Hall & 0.15 & Angew. Chem. Int. Ed. 2021, 60, 2 \\
\hline CuPc-pz COF & THz & 0.7 & J. Am. Chem. Soc. 2019, 141, 16810 \\
\hline CuPc-pz-I2 & THZ & $\sim 7$ & J. Am. Chem. Soc. 2020, 142, 21622 \\
\hline H2P-COF & FP-TRMC & 3.5 & Angew. Chem. Int. Ed. 2012, 51, 2618 \\
\hline CuPc-COF & FP-TRMC & 0.19 & Angew. Chem. Int. Ed. 2012, 51, 2618 \\
\hline NiPc-COF & FP-TRMC & 1.3 & Angew. Chem. Int. Ed. 2011, 50, 1289 \\
\hline 2D-NiPc-BTPA & FP-TRMC & 0.6 & J. Am. Chem. Soc. 2011, 133, 14510 \\
\hline HBC-COF & FP-TRMC & 0.7 & Nat. Commun. 2015, 6, 7786 \\
\hline
\end{tabular}


Table S4. Conductivity comparison of literature reported COFs.

\begin{tabular}{|c|c|c|c|}
\hline Sample & Method & Conductivity $\left(\mathrm{S} \mathrm{m}^{-1}\right)$ & Reference \\
\hline PAE-PcCu film & \multirow{3}{*}{ 2-Probe } & $3.8 \times 10^{-5}$ & \multirow{6}{*}{ This work } \\
\hline & & & \\
\hline PAE-PcCu film@I $\mathrm{I}_{2}$ & & 0.2 & \\
\hline PAE-PcCu film & \multirow{3}{*}{ Hall measurement } & $2.6( \pm 2.3) \times 10^{-5}$ & \\
\hline PAE-PcNi film & & $9.3( \pm 0.6) \times 10^{-5}$ & \\
\hline PAE-PcCo film & & $1.9( \pm 0.5) \times 10^{-4}$ & \\
\hline \multirow[t]{2}{*}{ TTF-COF } & \multirow{3}{*}{ 2-probe } & $1.2 \times 10^{-4}$ & \multirow{3}{*}{ Chem. Sci. 2014, 5, 4693} \\
\hline & & & \\
\hline TTF-COF@I $\mathrm{I}_{2}$ & & $2.8 \times 10^{-1}$ & \\
\hline CuPc-pz COF & \multirow{2}{*}{ Van der Pauw } & $3.3 \times 10^{-5}$ & \multirow{2}{*}{$\begin{array}{c}\text { J. Am. Chem. Soc. 2019, 141, } \\
16810\end{array}$} \\
\hline ZnPc-pz COF & & $7.0 \times 10^{-5}$ & \\
\hline \multirow[t]{2}{*}{ COF-DC-8 } & \multirow[t]{2}{*}{ 4-probe } & $2.51 \times 10^{-3}$ & \multirow{2}{*}{$\begin{array}{c}\text { J. Am. Chem. Soc. 2019, } 141 \\
11929\end{array}$} \\
\hline & & & \\
\hline \multirow{2}{*}{ TANG-COF } & \multirow{2}{*}{ 2-probe } & $5.0 \times 10^{-10}$ (in plane) & \multirow{2}{*}{$\begin{array}{c}\text { J. Am. Chem. Soc. 2020, 142, } \\
2155\end{array}$} \\
\hline & & $1.6 \times 10^{-3}$ (out-of-plane) & \\
\hline CuPc-pz COF@I2 & \multirow{2}{*}{ Van der Pauw } & $3.1 \times 10^{-2}$ & \multirow{2}{*}{$\begin{array}{c}\text { J. Am. Chem. Soc. 2020, 142, } \\
21622\end{array}$} \\
\hline ZnPc-pz COF@I2 & & $1.0 \times 10^{-2}$ & \\
\hline NiPc-CoTAA & 4-probe & $8.2 \times 10^{-3}$ & $\begin{array}{c}\text { Angew. Chem., Int. Ed. 2021, } \\
60,10806\end{array}$ \\
\hline
\end{tabular}




\section{References:}

1. Meng, Z.; Aykanat, A.; Mirica, K. A., Welding Metallophthalocyanines into Bimetallic Molecular Meshes for Ultrasensitive, Low-Power Chemiresistive Detection of Gases. J. Am. Chem. Soc. 2019, 141, 2046-2053.

2. Yang, C.; Dong, R.; Wang, M.; Petkov, P. S.; Zhang, Z.; Wang, M.; Han, P.; Ballabio, M.; Bräuninger, S. A.; Liao, Z.; Zhang, J.; Schwotzer, F.; Zschech, E.; Klauss, H.-H.; Cánovas, E.; Kaskel, S.; Bonn, M.; Zhou, S.; Heine, T.; Feng, X., A semiconducting layered metal-organic framework magnet. Nat. Commun. 2019, 10 (1), 3260-3270.

3. Ding, X.; Feng, X.; Saeki, A.; Seki, S.; Nagai, A.; Jiang, D., Conducting metallophthalocyanine 2D covalent organic frameworks: the role of central metals in controlling [small pi]-electronic functions. Chem. Commun. 2012, 48, 8952-8954.

4. Cai, S.-L.; Zhang, Y.-B.; Pun, A. B.; He, B.; Yang, J.; Toma, F. M.; Sharp, I. D.; Yaghi, O. M.; Fan, J.; Zheng, S.-R.; Zhang, W.-G.; Liu, Y., Tunable electrical conductivity in oriented thin films of tetrathiafulvalene-based covalent organic framework. Chem. Sci. 2014, 5 (12), 4693-4700.

5. Meng, Z.; Stolz, R. M.; Mirica, K. A., Two-Dimensional Chemiresistive Covalent Organic Framework with High Intrinsic Conductivity. J. Am. Chem. Soc. 2019, 141 (30), 11929-11937.

6. Kresse, G.; Furthmüller, J., Efficient iterative schemes for \textit\{ab initio\} total-energy calculations using a plane-wave basis set. Phys.Rev. B 1996, 54, 11169-11186.

7. Kresse, G.; Furthmüller, J., Efficiency of ab-initio total energy calculations for metals and semiconductors using a plane-wave basis set. Comput. Mater.Sci. 1996, 6, 15-50.

8. Perdew, J. P.; Chevary, J. A.; Vosko, S. H.; Jackson, K. A.; Pederson, M. R.; Singh, D. J.; Fiolhais, C., Atoms, molecules, solids, and surfaces: Applications of the generalized gradient approximation for exchange and correlation. Phys. Rev. B 1992, 46, 6671-6687.

9. Perdew, J. P.; Burke, K.; Ernzerhof, M., Generalized Gradient Approximation Made Simple. Phys. Rev. Lett. 1996, 77, 3865-3868.

10. Vladimir, I. A.; Aryasetiawan, F.; Lichtenstein, A. I., First-principles calculations of the electronic structure and spectra of strongly correlated systems: the LDA + U method. J. Phys. Condens. Matter 1997, 9 (4), 767-808.

11. Li, W.; Sun, L.; Qi, J.; Jarillo-Herrero, P.; Dinca, M.; Li, J., High temperature ferromagnetism in pi-conjugated twodimensional metal-organic frameworks. Chem Sci 2017, 8, 2859-2867.

12. Zhou, J.; Sun, Q., Magnetism of Phthalocyanine-Based Organometallic Single Porous Sheet. J. Am. Chem. Soc. 2011, $133,15113-15119$.

13. Lach, S.; Altenhof, A.; Tarafder, K.; Schmitt, F.; Ali, M. E.; Vogel, M.; Sauther, J.; Oppeneer, P. M.; Ziegler, C., MetalOrganic Hybrid Interface States of A Ferromagnet/Organic Semiconductor Hybrid Junction as Basis For Engineering Spin Injection in Organic Spintronics. Adv. Funct. Mater. 2012, 22, 989-997.

14. Monkhorst, H. J.; Pack, J. D., Special points for Brillouin-zone integrations. Physical Review B 1976, 13 (12), 5188 5192.

15. Bardeen, J.; Shockley, W., Deformation Potentials and Mobilities in Non-Polar Crystals. Phys. Rev. 1950, 80s, $72-80$.

16. Zhao, C.; Batiz, H.; Yasar, B.; Kim, H.; Ji, W.; Scott, M. C.; Chrzan, D. C.; Javey, A., Tellurium Single-Crystal Arrays by Low-Temperature Evaporation and Crystallization. Adv. Mater. 2021, 10.1002/adma.202100860.

17. Stoller, M. D.; Ruoff, R. S., Best practice methods for determining an electrode material's performance for ultracapacitors. Energy Environ. Sci. 2010, 3, 1294-1301.

18. Augustyn, V.; Come, J.; Lowe, M. A.; Kim, J. W.; Taberna, P.-L.; Tolbert, S. H.; Abruña, H. D.; Simon, P.; Dunn, B., High-rate electrochemical energy storage through Li+ intercalation pseudocapacitance. Nat. Mater. 2013, 12, $518-522$.

19. Biesinger, M. C.; Payne, B. P.; Grosvenor, A. P.; Lau, L. W. M.; Gerson, A. R.; Smart, R. S. C., Resolving surface 
chemical states in XPS analysis of first row transition metals, oxides and hydroxides: Cr, Mn, Fe, Co and Ni. Appl. Surf. Sci. 2011, 257 (7), 2717-2730.

20. DeBlase, C. R.; Silberstein, K. E.; Truong, T.-T.; Abruña, H. D.; Dichtel, W. R., $\beta$-Ketoenamine-Linked Covalent Organic Frameworks Capable of Pseudocapacitive Energy Storage. J. Am. Chem. Soc. 2013, 135, 16821-16824.

21. Zhang, L.; Zhang, F.; Yang, X.; Long, G.; Wu, Y.; Zhang, T.; Leng, K.; Huang, Y.; Ma, Y.; Yu, A.; Chen, Y., Porous 3D graphene-based bulk materials with exceptional high surface area and excellent conductivity for supercapacitors. Sci. Rep. 2013, 3, 1408-1417.

22. Halder, A.; Ghosh, M.; Khayum M, A.; Bera, S.; Addicoat, M.; Sasmal, H. S.; Karak, S.; Kurungot, S.; Banerjee, R., Interlayer Hydrogen-Bonded Covalent Organic Frameworks as High-Performance Supercapacitors. J. Am. Chem. Soc. 2018, 140, 10941-10945.

23. Izadi-Najafabadi, A.; Yasuda, S.; Kobashi, K.; Yamada, T.; Futaba, D. N.; Hatori, H.; Yumura, M.; Iijima, S.; Hata, K., Extracting the Full Potential of Single-Walled Carbon Nanotubes as Durable Supercapacitor Electrodes Operable at 4 V with High Power and Energy Density. Adv. Mater. 2010, 22, E235-E241.

24. Hao, L.; Ning, J.; Luo, B.; Wang, B.; Zhang, Y.; Tang, Z.; Yang, J.; Thomas, A.; Zhi, L., Structural Evolution of 2D Microporous Covalent Triazine-Based Framework toward the Study of High-Performance Supercapacitors. J. Am. Chem. Soc. 2015, 137, 219-225.

25. Sheberla, D.; Bachman, J. C.; Elias, J. S.; Sun, C.-J.; Shao-Horn, Y.; Dincă, M., Conductive MOF electrodes for stable supercapacitors with high areal capacitance. Nat. Mater. 2017, 16, 220-224. 